\title{
S/MIMO MC-CDMA Heuristic Multiuser Detectors Based on Single-Objective Optimization
}

\author{
Taufik Abrão • Leonardo D. de Oliveira • \\ Fernando Ciriaco • Bruno A. Angélico • \\ Paul Jean E. Jeszensky • Fernando Jose Casadevall Palacio
}

Published online: 15 April 2009

(C) The Author(s) 2009. This article is published with open access at Springerlink.com

\begin{abstract}
This paper analyzes the complexity-performance trade-off of several heuristic near-optimum multiuser detection (MUD) approaches applied to the uplink of synchronous single/multiple-input multiple-output multicarrier code division multiple access (S/MIMO MC-CDMA) systems. Genetic algorithm (GA), short term tabu search (STTS) and reactive tabu search (RTS), simulated annealing (SA), particle swarm optimization (PSO), and 1-opt local search (1-LS) heuristic multiuser detection algorithms (Heur-MuDs) are analyzed in details, using a single-objective antenna-diversity-aided optimization approach. MonteCarlo simulations show that, after convergence, the performances reached by all nearoptimum Heur-MuDs are similar. However, the computational complexities may differ
\end{abstract}

Part of this paper was presented at ISSSTA'08-The 10th International Symposium on Spread Spectrum Techniques and Applications, Bologna, Italy, Aug. 2008.

T. Abrão (ه)

Department of Electrical Engineering (DEEL), State University of Londrina (UEL), Londrina, PR 86051-990, Brazil

e-mail: taufik@uel.br

L. D. de Oliveira · F. Ciriaco · B. A. Angélico · P. J. E. Jeszensky

Department of Telecommunications and Control Engineering (LCS-PTC), Escola Politécnica of the University of São Paulo (EPUSP), São Paulo 05508-900, Brazil

e-mail: leonardo@1cs.poli.usp.br

F. Ciriaco

e-mail: fciriaco@lcs.poli.usp.br

B. A. Angélico

e-mail: angelico@1cs.poli.usp.br

P. J. E. Jeszensky

e-mail: pjj@1cs.poli.usp.br

F. J. Casadevall Palacio

Department of Signal Theory and Communication (TSC), Universitat Politècnica de Catalunya (UPC),

C. Jordi Girona 1-3, Campus Nord, Edificio D4, 08034 Barcelona, Spain

e-mail: ferranc@tsc.upc.edu 
substantially, depending on the system operation conditions. Their complexities are carefully analyzed in order to obtain a general complexity-performance framework comparison and to show that unitary Hamming distance search MUD (uH-ds) approaches (1-LS, SA, RTS and STTS) reach the best convergence rates, and among them, the 1-LS-MUD provides the best trade-off between implementation complexity and bit error rate (BER) performance.

Keywords MC-CDMA - S/MIMO - Multiuser detection · Heuristic methods · Tabu search · Genetic algorithm · Particle swarm optimization · Simulated annealing . Local search $\cdot$ Single-objective optimization $\cdot$ Computational complexity

\section{Introduction}

Multiuser detection (MUD) is a well known alternative to mitigate the multiple access interference (MAI) in a code division multiple access (CDMA) system [25,33]. The best performance is acquired by the optimum multiuser detection (OMUD), based on the log-likelihood function (LLF) [33]. However, this is achieved at the cost of huge computational complexity, which increases exponentially with the number of users. In the last decade, a variety of multiuser detectors with low complexity and sub-optimum performance were proposed, such as linear detectors [33], subtractive interference canceling [25], semidefinite programming approach by using interior-point methods [22,24,31,34], sphere decoder [14] and heuristic methods $[7,8,21,27,28]$. The last three methods have been used for solving different detection models and obtaining near-maximum likelihood (near-ML) performance at cost of polynomial computational complexity, except for the sphere decoder algorithm whose expected complexity increases exponentially according to the number of users for large size problems [14].

Multicarrier CDMA emerged from the combination of direct sequence CDMA (DSCDMA) and orthogonal frequency division multiplexing (OFDM) technologies [13]. While in DS-CDMA the spreading spectrum takes place in the time domain, in the classic MC-CDMA, the spreading is done in the frequency domain. Hence, the detector has the capacity to achieve frequency diversity at the cost of a reduced spreading factor. A modified MC-CDMA system that employs both time and frequency spreading is proposed in [35].

Multiuser reception under additive white Gaussian noise (AWGN) and/or selective frequency single-input single-output (SISO) channels using local search, particle swarm optimization and simulated annealing based detectors has been studied earlier $[16,26,27,38]$ and shown to have excellent near-optimum performance at low to moderate complexity. Recently, heuristic algorithms have been applied to symbol detection in non-spreading multiple-input multiple-output systems [4,24]. PSO heuristic method has been applied to non-spreading MIMO multiuser detection on 16- and 64-QAM modulation [19,37]. On the other hand, there are few works applying heuristic sub-optimal multiuser detection approaches to MIMO MC-CDMA systems. In [32], a large S/MIMO MC-CDMA system ${ }^{1}$ with sub-optimal nearexponential diversity performance and low-complexity, based on a kind of directional search, was proposed. Essentially, the algorithm searches out a sequence of bit vectors on a monotonic likelihood ascent, and converges to a fixed point, within a finite number of steps [32]. A survey on MIMO OFDM systems, focused on multiuser detection and estimation heuristics approaches, is provided in [15].

This work aims to evaluate the performance of several heuristic multiuser detectors for synchronous S/MIMO MC-CDMA systems, taking into account their computational complexity.

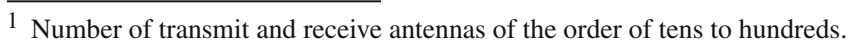


In particular, GA, STTS, RTS, SA, PSO and 1-LS algorithms are considered in a singleobjective antenna-diversity-aided optimization. In this sense, the contributions of this paper are two-fold: (a) provide a general framework analysis for heuristic multiuser detectors approaches suitable for S/MIMO MC-CDMA systems, since there are no comparative studies available in the literature; (b) offer evidences for the feasibility of low-complexity uH-ds MUD approaches, specially for the 1-LS multiuser detector applied to MC-CDMA systems with low order modulation and flat fading S/MIMO channels.

The paper is organized as follows. The system model and single-objective optimization approach are described in Sect. 2. A detailed description of heuristic assisted MUD for S/MIMO MC-CDMA system, addressing input parameters choices and pseudo-codes for the implemented algorithms, is provided in Sect.3. Section 4 analyzes the computational complexity in terms of necessary floating point operations for each MUD to achieve convergence and presents some complexity indexes. The numerical results, including performance under channel estimation errors and complexity evaluation, are discussed in Sect. 5. The main conclusions are pointed out in Sect. 6.

\section{System Model}

The MC-CDMA transmitter shown in Fig. 1 employs both time- and frequency-domain spreading. The information bit of the $k$ th user with duration $T_{b}$ is spread in $M$ parallel subcarriers. On each subcarrier, the resultant signal is time-domain spread by a sequence $c_{k, m}(t), m=0, \ldots, M-1$, with $N$ chips of $T_{c}$ duration, such that $N=T_{b} / T_{c}$. The $k$ th

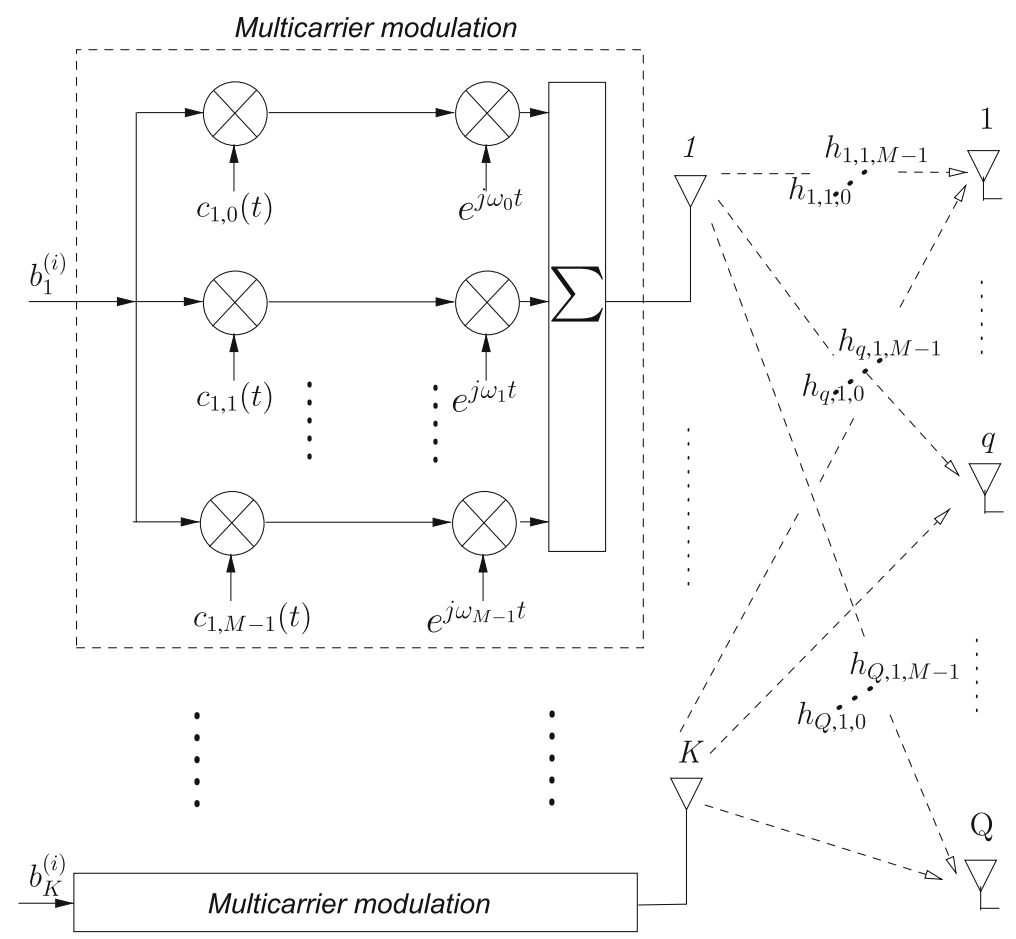

Fig. 1 Equivalent S/MIMO MC-CDMA transmitter scheme 
user's transmitted signal has the form

$$
s_{k}(t)=\sqrt{\frac{2 E_{b k}}{M}} \sum_{i=-\infty}^{\infty} \sum_{m=0}^{M-1} c_{k, m}(t) b_{k}^{(i)} e^{J \omega_{m} t},
$$

where $E_{b k}$ is the $k$ th signal energy per bit and $b_{k}^{(i)} \in[-1,1]$ is the $i$ th transmitted bit related to the $k$ th user. The spreading sequence signed to the $m$ th subcarrier of the $k$ th user can be expressed by

$$
c_{k, m}(t)=\sum_{n=0}^{N-1} c_{k, m}^{(n)} p\left(t-n T_{c}\right)
$$

with $c_{k, m}^{(n)} \in[-1,1]$, and $p(t)$ being a rectangular pulse shape. Once the signature waveform $c_{k, m}(t)$ is used to spread the data bits to $N$ chips in the time-domain and to map them into a total of $M$ subcarriers in the frequency-domain for all $K$ users, then the total processing gain is $N M$. Additionally, it is assumed that the signature waveforms have normalized energy, $\int_{0}^{T_{b}} c_{k, m}^{2}(t) d t=1, \forall k, m$.

The synchronous MC-CDMA system has $K$ mobile users equipped with single-antenna terminals and a base station with $Q$ receive antennas, characterizing a SIMO channel. Equivalently, the model could describe a MIMO MC-CDMA communication system for a single-user equipped with $K$ transmit antennas and $Q$ receive antennas. Model extension for asynchronous MC-CDMA systems is straightforward and can be found in [39].

In antenna-diversity-aided systems, the antennas are assumed to be sufficiently separated such that the received signals at each element are faded independently, resulting in an independent LLF for each antenna. Hence, this model assumes an equivalent independent Rayleigh flat channel on each subcarrier over all $Q$ receive antennas. For the $k$ th user, the channel impulse response on the $m$ th subcarrier of the $q$ th receive antenna is given by

$$
h_{q, k, m}^{(i)}=\beta_{q, k, m}^{(i)} e^{J \varphi_{q, k, m}^{(i)}}
$$

where the amplitude $\beta_{q, k, m}^{(i)}$ is a Rayleigh distributed random variable and the phase $\varphi_{q, k, m}^{(i)}$ is uniformly distributed in the $[0,2 \pi)$ interval, assumed to be constants during the $i$ th information bit. The signal received on the $q$ th receive antenna, $m$ th subcarrier, from all $K$ users, is given by

$$
r_{q, m}(t)=\sum_{i=-\infty}^{\infty} \sum_{k=1}^{K} \sqrt{\frac{2 E_{b k}}{M}} b_{k}^{(i)} c_{k, m}\left(t-i T_{b}\right) \beta_{q, k, m}^{(i)} e^{J\left(\omega_{m} t+\varphi_{q, k, m}^{(i)}\right)}+\eta(t),
$$

where $\eta(t)$ is the AWGN term associated to the $m$ th subcarrier bandwidth of $q$ th antenna and with bilateral power spectral density given by $N_{0} / 2$. For simplicity, from now on, the index $i$ will be dropped.

The receiver structure is shown in Fig. 2. For each receive antenna of each user, the signal is demodulated in all $M$ subcarriers and passed through a matched filter bank (MFB), generating decision variables. The resulting signal is submitted to a heuristic-assisted MUD described in Sect. 3. Notice that the channel state information (CSI) has to be estimated at the receiver side by either training or some blind method.

The equivalent baseband representation of the received signal on the $m$ th subcarrier of the $q$ th antenna, $\mathrm{r}_{q, m}(t)$, can be expressed in matrix notation

$$
\mathbf{r}_{q, m}(t)=\mathbf{C}_{m} \mathbf{W}_{q, m} \mathbf{b}+\mathbf{n}_{q, m},
$$




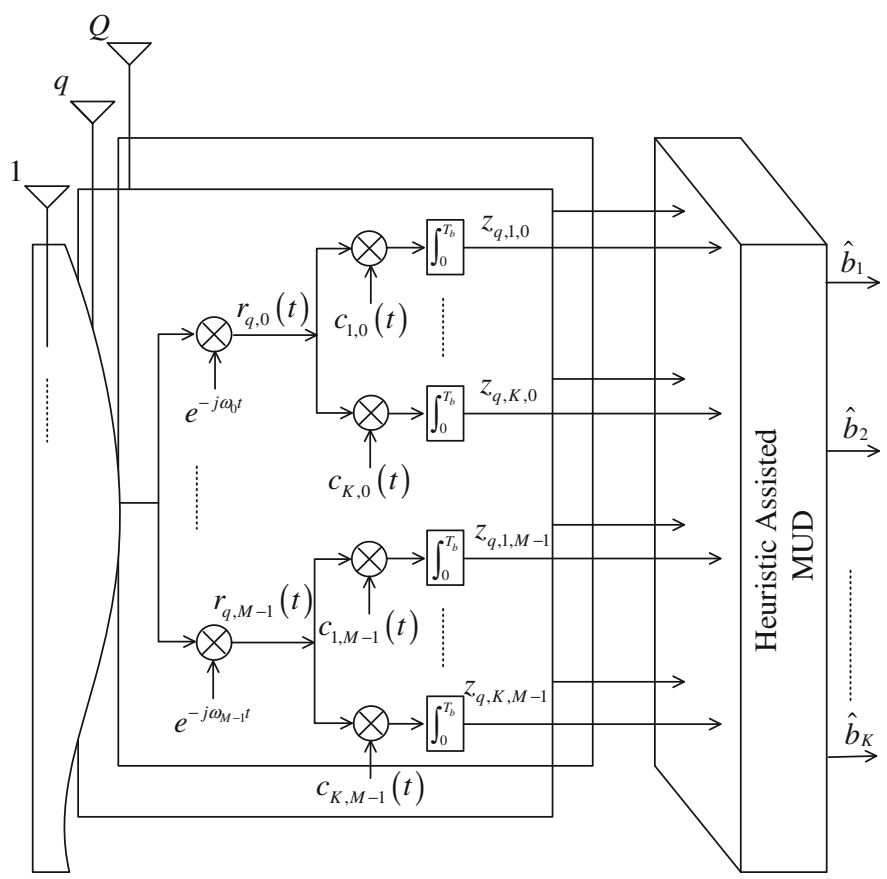

Fig. 2 MC-CDMA receiver structure considering $Q$ receive antennas

with $\mathbf{C}_{m}=\left[\mathbf{c}_{1, m} \ldots \mathbf{c}_{k, m} \ldots \mathbf{c}_{K, m}\right], \mathbf{c}_{k, m}=\left[c_{k, m}^{(0)} \ldots c_{k, m}^{(N-1)}\right]^{\top}, \mathbf{W}_{q, m}=\operatorname{diag}\left[A_{1} h_{q, 1, m}\right.$ $\left.\ldots A_{k} h_{q, k, m} \ldots, A_{K} h_{q, K, m}\right], A_{k}=\sqrt{\frac{E_{b k}}{M}}, \mathbf{b}=\left[b_{1} \ldots b_{k} \ldots b_{K}\right]^{\top}, \mathbf{n}_{q, m}$ being the $N-$ sampled AWGN, and $(\cdot)^{\top}$ representing the transpose operator. A mathematical expression of the MFB output on the $m$ th subcarrier, $q$ th antenna, without phase compensation, can be represented in the vector notation

$$
\mathbf{z}_{q, m}=\left[z_{q, 1, m} \ldots z_{q, k, m} \ldots z_{q, K, m}\right]^{\top}=\mathbf{R}_{m} \mathbf{W}_{q, m} \mathbf{b}+\tilde{\mathbf{n}}_{q, m},
$$

where $\tilde{\mathbf{n}}_{q, m}=\left[\tilde{n}_{q, 1, m} \ldots \tilde{n}_{q, k, m} \ldots \tilde{n}_{q, K, m}\right]$ is the filtered noise vector, and $\mathbf{R}_{m}$ is the correlation matrix

$$
\mathbf{R}_{m}=\left[\begin{array}{cccc}
1 & \rho_{1,2}^{(m)} & \cdots & \rho_{1, K}^{(m)} \\
\rho_{2,1}^{(m)} & 1 & \cdots & \rho_{2, K}^{(m)} \\
\vdots & \vdots & \ddots & \vdots \\
\rho_{K, 1}^{(m)} & \rho_{K, 2}^{(m)} & \cdots & 1
\end{array}\right],
$$

with the auto $(i=j)$ and cross-correlation $(i \neq j)$ of the spreading code being defined by $\rho_{i, j}^{(m)}=\int_{0}^{T_{b}} c_{i, m}(t) \cdot c_{j, m}(t) d t$. Considering the maximum ratio combining (MRC) rule, the decision variables for each receive antenna are obtained from (6) as 


$$
\breve{\mathbf{z}}_{q, m}=\left[\breve{z}_{q, 1, m} \ldots \breve{z}_{q, k, m} \ldots \breve{z}_{q, K, m}\right]^{\top}=\widehat{\mathbf{W}}_{q, m}^{*} \mathbf{R}_{m} \mathbf{W}_{q, m} \mathbf{b}+\breve{\mathbf{n}}_{q, m},
$$

where $\breve{\mathbf{n}}_{q, m}$ is the AWGN weighted by complex combination coefficients, $\widehat{\mathbf{W}}_{q, m}$ is an estimate of $\mathbf{W}_{q, m}$, and ${ }^{*}$ represents the conjugate complex operator. Hence, for the $k$ th user bit estimation, the conventional detector (CD) linearly combines decision variables over all $M$ subcarriers and $Q$ receive antennas

$$
b_{k}^{\mathrm{cMFB}}=\operatorname{sign}\left(\sum_{q=1}^{Q} \sum_{m=0}^{M-1} \Re\left\{\breve{z}_{q, k, m}\right\}\right), \quad k=1, \ldots, K,
$$

with $\mathbf{b}^{\mathrm{cMFB}}=\left[b_{1}^{\mathrm{cMFB}} \ldots b_{K}^{\mathrm{cMFB}}\right]^{\top}, \Re\{\cdot\}$ representing the real operator, and

$$
\operatorname{sign}(x)=\left\{\begin{array}{ll}
-1 & \text { if } x<0 \\
+1 & \text { if } x \geq 0
\end{array} .\right.
$$

In the presence of diversity, the multiuser detection optimization problem can be modeled using a single or a multi-objective function. The present work considers only a linear combination single-objective antenna-diversity-aided strategy (LC Q-LLFs).

At the receiver, the maximum likelihood detector jointly detects the data of all users and minimizes the MAI effects. The optimum multiuser detection for S/MIMO MC-CDMA is based on the maximum likelihood criterion [33], that chooses a candidate $\mathbf{b}_{i}$ which maximizes

$$
\widehat{\mathbf{b}}^{\mathrm{opt}}=\arg \left\{\max _{\mathbf{b}_{i} \in \mathcal{A}^{\mathcal{M} K}}\left[f\left(\mathbf{b}_{i}\right)\right]\right\},
$$

where $\mathcal{M}$ is the message length and $\mathcal{A}$ is the dimension of symbol alphabet. In the optimization context, $\mathbf{b}_{i}$ is a vector-candidate and $\mathcal{A}^{\mathcal{M} K}$ is the feasible region in the decision space, and the objective function, $f\left(\mathbf{b}_{i}\right)$, considers a combination rule over $M$ subcarriers and $Q$ receive antenna signals, such that

$$
f\left(\mathbf{b}_{i}\right)=\frac{1}{Q} \sum_{q=1}^{Q} \sum_{m=0}^{M-1} \Omega_{q, m}\left(\mathbf{b}_{i}\right)
$$

with [33]

$$
\Omega_{q, m}\left(\mathbf{b}_{i}\right)=2 \Re\left\{\mathbf{b}_{i}^{\top} \widehat{\mathbf{W}}_{q, m}^{*} \mathbf{z}_{q, m}\right\}-\mathbf{b}_{i}^{\top} \widehat{\mathbf{W}}_{q, m} \mathbf{R}_{q, m} \widehat{\mathbf{W}}_{q, m}^{*} \mathbf{b}_{i} .
$$

The evaluation in (10) can either be extended along the whole message, where all symbols of the transmitted vector for all $K$ users are jointly detected (vector ML approach) or the decisions can be taken considering the optimal single symbol detection of all $K$ multiuser signals (symbol ML approach). This work considers only the symbol ML detection approach, i.e, $\mathcal{M}=1$.

Equation(10) is a combinatorial optimization problem, which requires an exhaustive search in $\mathcal{A}^{\mathcal{M} K}$ possibilities of $\mathbf{b}_{i}$. Thus, in order to obtain a lower complexity detector, this paper considers near-optimum detector schemes that search for a solution in a space $\mathcal{S}$, smaller than the entire space $\left(\mathcal{S} \subset \mathcal{A}^{\mathcal{M} K}\right.$ ), and have a high probability of finding the optimal solution (i.e., correct symbol detection). 


\section{Single-Objective Heur-MuD for S/MIMO MC-CDMA}

In the LC Q-LLFs vector-candidate-selection strategy, the choice of vector-candidate(s) for evolution is based on the highest fitness values of (11) and decisions are based on a single-objective optimization procedure, i.e., by combining the subcarriers and antenna-specific performance measures. Other works considering LC Q-LLFs evaluation in a multiuser detection problem include [4,36]. Different aspects of PSO have been analyzed previously in [3]. Descriptions, details, pseudo-codes, as well as performance-complexity analysis for all HeurMuDs, are provided in the sequel.

\subsection{Genetic MuD for S/MIMO MC-CDMA}

Genetic algorithms are based on selection mechanism and natural evolution, following Darwin's theory of species' evolution, which explains the history of life through the action of physical processes and genetic operators in populations or species. These processes, known as reproduction, perturbation, competition and selection, are responsible for an efficient search for the global solution. In GA, each individual represents a vector-candidate.

In the GA applied to MUD, the population size is chosen in the initialization stage and maintained constant in all generations (iterations). The aptitude is measured through the LC Q-LLF (11) and it is directly responsible for death or life of individuals. The CD vector estimates, $\mathbf{b}^{\mathrm{cMFB}}$, is adopted as the initial GA's individual. The other $(\mathcal{P}-1)$ members of the first population are obtained from the initial individual with convenient perturbations [7].

The selection process chooses the best $T$ individuals from the population $\mathcal{P}$ as parents for the next generation. The mating pool size, $T$, has to be selected in the interval $2 \leq T<\mathcal{P}$, in order to accomplish a good convergence velocity and final solution [12,23]. For this MUD problem, a fixed mating pool size $T=\max \{2, \mathcal{P} / 10\}$ is adopted.

For the genetic operators, this work adopts the uniform crossover [12,23] with crossover probability $p_{c}$, and mutation based on noise, such that

$$
\mathcal{Z}_{p_{m}}\left\{\mathbf{b}_{i}\right\}=\operatorname{sign}\left[\mathbf{b}_{i}+\mathcal{N}\left(0, \sigma^{2}\right)\right],
$$

where $\mathcal{Z}_{p_{m}}$ is the mutation operator over $\mathbf{b}_{i}$ individual and $\mathcal{N}\left(0, \sigma^{2}\right)$ represents a Gaussian distribution with zero mean and standard deviation $\sigma$, which is related with the mutation probability $p_{m}[2,7]$.

The GA-MUD uses a replacement strategy called global elitism [23], where only the best $\mathcal{P}$ individuals from the joint population of parents and offspring are maintained for the next generation. Finally, the optimization process finishes after a fixed number of generations $(G)$.

Algorithm 1 in Appendix describes the implemented GA-MUD for S/MIMO MC-CDMA system.

\subsection{Simulated Annealing MUD for S/MIMO MC-CDMA}

The simulated annealing algorithm concept stems from thermal annealing, which aims to obtain perfect crystallizations by a sufficiently slow temperature reduction to give atoms the time to attain the lowest energy state $[6,20,30]$. In order to escape from a local solution, the SA algorithm uses an acceptance probability function, proportional to the temperature, which allows it to accept a particular solution having a higher value (a state of higher energy). This mechanism makes possible for the algorithm to leave a local solution area and to seek for the global solution in other areas. 
Classically, the acceptance probability function is inspired by the Boltzman distribution

$$
P(\Delta E)=\exp \left(\frac{\Delta E}{T_{k}}\right),
$$

where $\Delta E=f\left(\mathbf{b}_{\mathbf{i}}^{\prime}\right)-\mathbf{f}\left(\mathbf{b}_{\mathbf{i}}\right), \mathbf{b}_{\mathbf{i}}^{\prime}$ is a set of possible received bits that differs from $\mathbf{b}_{i}$ in only one bit, i.e., it is a unitary Hamming distance from $\mathbf{b}_{i}$, and $T_{k}$ is the temperature in the current iteration, defined by

$$
T_{k}=\delta^{k} T_{0}
$$

with $T_{0}$ being the initial temperature of the process, and $\delta$ the cooling rate. The initial temperature of the process has a high value, $T_{0}>>0$, and this temperature is reduced after a fixed number of iterations It . Hence, after each It iterations, $k=k+1$. Equation (15) is not the only way to describe the cooling process, but one of the usual methods found in literature $[6,17]$.

Besides the initial set of received bits $\mathbf{b}^{\mathrm{cMFB}}$ and the number of iterations $G$, the SA-MUD must be initialized with three more parameters: $T_{0}, \delta$, and $I t$, which were empirically adjusted through a non-exhaustive attempt procedure [29].

Algorithm 2 in Appendix describes the implemented SA-MUD for S/MIMO MC-CDMA system.

\subsection{Short Term Tabu Search MuD for S/MIMO MC-CDMA}

STTS is based on a deterministic operation way of memory. The memory is implemented through recording displacement characteristics of the previously visited solutions [9-11,30], avoiding cycling. This is described by the tabu list (TL), which is formed by the search's recent past, being called effect of short term memory. Those displacement characteristics are forbidden on the tabu list for a certain number of iterations. This helps to avoid returns to local solutions, promoting diversification in the search. The pseudo-code of the STTS-MUD for S/MIMO MC-CDMA system is described in Appendix, Algorithm 3.

\subsection{Reactive Tabu Search MuD for S/MIMO MC-CDMA}

RTS combines the effect of short term with another memory effect to avoid returns to local solution and to provide an efficient search. This effect is known as long term memory, which alternates between intensification and diversification of the search [5,30].

The tabu list of the STTS algorithm is implemented using the effects of short term memory. However, it does not avoid returns to local solution. Additionally, the choice of a fixed prohibition period $\left(P_{T L}\right)$ appropriated for each problem, becomes a difficult task, because a small period is insufficient to prevent returns to local solutions and an excessively long period reduces the amount of possible displacements, resulting in an inefficient search.

RTS long-term memory is constituted by the effect of short term memory of the STTS algorithm, but adapting its tabu list prohibition period during the search, assuming that this period varies in each iteration $\left(P_{T L}^{g}\right)$. The prohibition period is initialized with a small value, $P_{T L}^{0}$, which is adapted when repetitions take place. If a repetition is found, diversification is encouraged by incrementing $P_{T L}^{g}$. For that diversification not to assume very high values after some iterations, the period $P_{T L}^{g}$ is reduced when $\left|f\left(\mathbf{b}_{i}\right)-f_{\text {best }}^{g}\right|<x f_{\text {best }}^{g}, \forall i$, where $f_{\text {best }}^{g}$ is the best solution (cost function or energy value) until the $g$ th iteration, and $0<x<1$. 
A robust value for the constant that controls the reduction of $P_{T L}^{g}$ was experimentally obtained in [30], and $x=0.3$ was found for the AWGN MUD problem.

The RTS algorithm is very similar to STTS, except for the input parameters and the step 2.f, Algorithm 3 in Appendix, that has to be substituted by the steps given in Algorithm 4.

\subsection{Local Search MUD for S/MIMO MC-CDMA}

The local search is an optimization method that consists of searches in a previously established neighborhood [1]. It is important to restrict the neighborhood and to choose a good initial vector-candidate in order to find a valid solution with low complexity. In this work it is adopted the 1-LS algorithm, which seeks the solution within a unitary Hamming distance space from the vector-solution so far, i.e., the search is done over vectors that differ in only one bit from the current solution. The combined matched filter outputs, $\mathbf{b}^{\mathrm{cMFB}}$, is taken as the best initial solution [26].

All the $K$ vectors with unitary Hamming distance to the current best solution are evaluated through the cost function computation, (11). The vector-candidate that results in the largest value for the cost function is selected, and if it is better than the previous one, it is taken as the new best solution and a new iteration is performed; otherwise, the search is concluded. The stop criterion can be seen as the absence of improvement in one iteration.

Three advantages make the local search algorithm a natural choice for the MuD problem: (a) absence of input parameters; (b) simple stop criterion, avoiding prior calculation; (c) simple strategy.

The steps to implement the 1-LS-MUD for S/MIMO MC-CDMA system are described in Appendix, Algorithm 5.

\subsection{Particle Swarm Optimization MuD for S/MIMO MC-CDMA}

The PSO principle is the movement of a group of particles, randomly distributed in the search space, each one with its own position and velocity. The position of each particle is modified by the application of velocity in order to reach a better performance [18]. In PSO, a particle represents a vector-candidate, with a position at instant (iteration) $t$, given by

$$
\mathbf{b}_{i}[\mathrm{t}]=\left[b_{i 1}[\mathrm{t}] \ldots b_{i k}[\mathrm{t}] \ldots b_{i K}[\mathrm{t}]\right]^{\top} .
$$

The particle(s) selection for evolving is based on the highest fitness values in (11). The interaction among particles is inserted in the calculation of the particles velocity. For the LC Q-LLF selection strategy, the $i$ th particle velocity, $\mathbf{v}_{i}[\mathrm{t}]$, is given by

$$
\mathbf{v}_{i}[\mathrm{t}+1]=\omega \mathbf{v}_{i}[\mathrm{t}]+\phi_{1} \mathbf{U}_{i_{1}}[\mathrm{t}]\left(\mathbf{b}_{i}^{\text {best }}[\mathrm{t}]-\mathbf{b}_{i}[\mathrm{t}]\right)+\phi_{2} \mathbf{U}_{i_{2}}[\mathrm{t}]\left(\mathbf{b}_{g}^{\text {best }}[\mathrm{t}]-\mathbf{b}_{i}[\mathrm{t}]\right),
$$

where $\omega$ is the inertial weight; $\mathbf{U}_{i_{1}}[\mathbf{t}]$ and $\mathbf{U}_{i_{2}}[\mathbf{t}]$ are diagonal matrices with dimension $K$, whose elements are random variables uniformly distributed in the $[0,1]$ interval, generated for the $i$ th particle at instant $\mathbf{t} ; \mathbf{b}_{g}^{\text {best }}[\mathbf{t}]$ and $\mathbf{b}_{i}^{\text {best }}[\mathrm{t}]$ are the best global position and the best local positions found until the iteration $\mathrm{t}$, respectively; $\phi_{1}$ and $\phi_{2}$ are the individual and global acceleration coefficients, respectively. Typical values of these parameters are $\phi_{1}=2$ and $\phi_{2}=2[18]$.

PSO adopts the selection of only one $\mathbf{b}_{i}^{\text {best }}[\mathrm{t}]$ for each particle and only one $\mathbf{b}_{g}^{\text {best }}[\mathrm{t}]$ for the entire population, obtained through the linear combination signals over $Q$ antennas.

For the MUD optimization problem with binary modulation, each element $b_{i k}[\mathrm{t}]$ in (17) just assumes " 0 " or " 1 " values. Hence, a discrete mode for the position choice is carried out 
Table 1 Minimum bit change probability as a function of $V_{\max }$

\begin{tabular}{llllll}
\hline$V_{\max }$ & 1 & 2 & 3 & 4 & 5 \\
\hline $1-S\left(V_{\max }\right)$ & 0.2690 & 0.1192 & 0.0474 & 0.0180 & 0.0067 \\
\hline
\end{tabular}

inserting a probabilistic decision step based on threshold, depending on the velocity. Several functions have this characteristic, such as the sigmoid function: $S\left(v_{i k}[\mathrm{t}]\right)=\left\{1+e^{-v_{i k}[\mathrm{t}]}\right\}^{-1}$ where $v_{i k}[\mathrm{t}]$ is the $k$ th element of the $i$ th particle velocity. This function is limited in the $[0,1]$ interval. Therefore, in this work, the selection of the future particle position is obtained through the statement

$$
\begin{aligned}
& \text { if } v_{i k}[\mathrm{t}]<S\left(v_{i k}[\mathrm{t}]\right), \\
& \quad b_{i k}[\mathrm{t}+1]=1, \\
& \text { otherwise, } \\
& \quad b_{i k}[\mathrm{t}+1]=0,
\end{aligned}
$$

where $v_{i k}[\mathrm{t}]$ is a random variable with uniform distribution in the $[0,1]$ interval.

The population size is maintained constant in all iterations. In order to obtain further diversification for the search universe, a factor $\left(V_{\max }\right)$ is added to the PSO model, which is responsible for limiting the velocity in the range $\left[ \pm V_{\max }\right]$, allowing the algorithm to escape from a possible local solution. Some probabilities of bit change when the particle velocity crosses the limits established by $\left[ \pm V_{\max }\right]$ are shown in Table 1 .

After the search has finished ( $G$ iterations), the estimated vector is calculated as: $\widehat{\mathbf{b}}=$ $\mathbf{b}_{\mathrm{g}}^{\text {best }}[G]$, which is associated with the particle position that maximizes (11).

Algorithm 6 in Appendix describes the implemented PSO-MUD for S/MIMO MC-CDMA system.

\section{Computational Complexity}

Although the Heur-MUD schemes have similar performances, the number of operations varies according to the strategy. This work takes into account the number of floating point operations necessary for achieving convergence, defined here as computational complexity. The considered operations are: multiplication, comparison and random number generation. This analysis is limited by the fact that operations of distinct computational complexity are considered as having the same cost. The complexity is expressed as a function of the number of users $(K)$, receivers $(Q)$, subcarriers $(M)$, iterations needed for convergence $(\mathcal{G} \leq G)$, Tabu prohibition period $\left(P_{T L}\right.$ and $\left.\left\langle P_{T L}^{m}\right\rangle\right)$, and population size $(\mathcal{P})$. The total number of operations $C$ for all heuristic multiuser detectors and OMUD is summarized in Table 2 .

The cost function calculation in (11) is the most significant factor in determining the complexity of the detectors. The terms $\widehat{\mathbf{W}}_{q, m} \mathbf{R}_{q, m} \widehat{\mathbf{W}}_{q, m}^{*}$ and $\widehat{\mathbf{W}}_{q, m}^{*} \mathbf{z}_{q, m}$ are evaluated outside the iterations loop and are constant during the detection search. The algorithms perform $4 K^{3}+K^{2}$ operations on those two terms, which are calculated $Q M$ times. Inside each loop, the number of operations needed for evaluating a vector-candidate through the cost function becomes $Q M\left(K^{2}+2 K\right)$. Thus, for reference, the complexity to evaluate the cost function once (one-CFC) is included in Table 2. 
Table 2 Computational complexity $\mathrm{C}$ for the Heur-MuDs

\begin{tabular}{ll}
\hline MuD & Operations \\
\hline OMuD & $2^{K} Q M\left(K^{2}+2 K\right)+Q M\left(4 K^{3}+K^{2}\right)$ \\
GA & $Q M\left[K^{2}(4 K+1)+\mathcal{P} K(\mathcal{G}+1)(K+2)\right]$ \\
& $\quad+\mathcal{P}[K(8.5 \mathcal{G}+2)+4(\mathcal{G}+1)]-(K+3 \mathcal{P})$ \\
SA & $Q M\left[K^{3}(\mathcal{G}+5)+2 K^{2}(\mathcal{G}+1)\right]+K \mathcal{G}\left(4+\frac{7}{K}+\frac{M}{\mathcal{G}}\right)$ \\
STTS & $Q M\left[K^{3}(\mathcal{G}+5)+2 K^{2}(\mathcal{G}+1)\right]$ \\
& $\quad+K \mathcal{G}\left(5+2 K+P_{T L}+\frac{9}{K}+\frac{M}{\mathcal{G}}\right)$ \\
& $\quad Q M\left[K^{3}(\mathcal{G}+5)+2 K^{2}(\mathcal{G}+1)\right]$ \\
RTS & $\quad+K \mathcal{G}\left(5+2 K+\left\langle P_{T L}^{m}\right\rangle+\frac{13}{K}+\frac{M}{\mathcal{G}}\right)$ \\
& $\quad \mathcal{G}\left[Q M K\left(K^{2}+2 K\right)+2 K+1\right]+Q M\left(4 K^{3}+2 K^{2}+2 K\right)$ \\
1-LS & $\quad \mathcal{G}\left[Q M \mathcal{P}\left(K^{2}+2 K\right)+9 \mathcal{P} K+2 \mathcal{P}+1\right]$ \\
PSO & $\quad Q M\left(4 K^{3}+K^{2}\right)+(\mathcal{P}-1) K$ \\
one-CFC & $2 Q M K\left(2 K^{2}+K+1\right)$ \\
\hline
\end{tabular}

Essentially, the difference in terms of computational complexity among the Heur-MuDs lies on the generation of the new vector-candidate populations for each search iteration. Hence, for 1-LS, the cost function is evaluated $K$ times in each iteration and there are also $\mathcal{G}(K+1)$ multiplications and $\mathcal{G}(K+1)+1$ comparisons. For PSO, $\mathcal{P}$ cost functions are performed in each iteration, and $3 \mathcal{G} \mathcal{P} K+(\mathcal{P}-1) K$ random number generations, $\mathcal{G}(3 \mathcal{P} K+2 \mathcal{P}+1)$ comparisons and $\mathcal{G}(3 \mathcal{P} K+1)$ multiplications are required. In each SA iteration, $K$ cost functions are evaluated; there are also $\mathcal{G}(3 K+1)$ multiplications, $\mathcal{G}(K+5)$ comparisons and $\mathcal{G}$ random number generations. For GA, $\mathcal{P}$ cost functions are performed in each iteration, and $3 \mathcal{P G}+\mathcal{P}-1$ bit generations, $T \mathcal{G}$ selections, $3 \mathcal{P G}$ ordinations, $\mathcal{P G}$ comparisons and $\mathcal{P G}$ changes of bits are required. For STTS, $\mathcal{G} K+1$ cost functions, $\mathcal{G} K$ changes of bits, $\mathcal{G} K$ bit generations and $4 \mathcal{G} K+\mathcal{G} K P_{T L}$ comparisons are performed. Finally, for RTS, $\mathcal{G} K+1$ cost functions, $\mathcal{G} K$ changes of bits, and $4 \mathcal{G} K+\mathcal{G} K\left\langle P_{T L}^{m}\right\rangle$ comparisons are performed, where $\left\langle P_{T L}^{m}\right\rangle=\frac{1}{\mathcal{G}} \sum_{g=1}^{\mathcal{G}} P_{T L}^{g}$.

In addition, the complexities of the Heur-MuDs are numerically evaluated in Sect.5.3 considering two figures of merit, the complexity factor $(\mathrm{CF})$ and the complexity reduction (CR) indexes,

$$
\mathrm{CF}_{\text {Heur }}=\frac{\mathrm{C}_{\text {Heur }}}{\text { one-CFC }} \text { and } \mathrm{CR}_{\text {Heur }}=\frac{\mathrm{C}_{\text {Heur }}}{\mathrm{C}_{\mathrm{OMUD}}},
$$

where $\mathrm{C}_{\mathrm{Heur}}$ is the computational complexity needed for each Heur-MUD to converge, $\mathrm{COMUD}$ is the complexity of the OMUD, and one-CFC is the computational complexity of one cost function calculation, presented in Table 2 .

\section{Numerical Results and Discussion}

The BER performance results are obtained as a function of the signal-to-noise ratio (SNR, or equivalently $E_{b} / N_{0}$ ), the iterations (generations) needed for convergence, and the number of users (or system loading, $L=\frac{K}{N M}$ ). A perfect power control is assumed, which means that $E_{b k}=E_{b}\left(A_{k}=A\right)$. The main system and channel parameters used in this section are summarized in Table 3. This paper adopts random generated sequences of length 32 . The 
Table 3 System, algorithm and channel parameters

\begin{tabular}{|c|c|}
\hline Parameter & Adopted values \\
\hline \multicolumn{2}{|l|}{ S/MIMO MC-CDMA System } \\
\hline \# Rx antennas & $Q=1-3$ \\
\hline Code sequences & Random with length 32 \\
\hline Processing gain & $N=8$ \\
\hline Subcarriers & $M=4$ \\
\hline Bit period, $T_{b}$ & $300 \mu \mathrm{s}$ \\
\hline \# mobile users & $K=2 ; 5 ; 10 ; 15 ; 20 ; 25 ; 32$ \\
\hline Received SNR & $E_{b} / N_{0} \in[0 ; 18] \mathrm{dB}$ \\
\hline \multicolumn{2}{|l|}{ All Heur-MuDs } \\
\hline \# iterations & $G \in[3 ; 40]$, depending on $(K, Q)$ \\
\hline \multicolumn{2}{|l|}{ GA parameters } \\
\hline GA pop. size & $\mathcal{P}=30$ vector-candidates \\
\hline Mutation probability & $p_{m}=1 / K$ \\
\hline Crossover probability & $p_{c}=0.5$ \\
\hline Mating pool size & $T=\max \{2, \mathcal{P} / 10\}$ \\
\hline \multicolumn{2}{|l|}{ SA parameters } \\
\hline \# iterations @ $T_{k}$ & $I t=2$ \\
\hline Initial Temperature & $T_{0}=0.3 K$ \\
\hline Cooling rate & $\delta=0.9$ \\
\hline \multicolumn{2}{|l|}{ STTS parameters } \\
\hline $\begin{array}{l}\text { Tabu list length } \\
\text { RTS parameters }\end{array}$ & $P_{T L}=3$ \\
\hline $\begin{array}{l}\text { Tabu list: initial \& final length } \\
\text { Reduction control }\end{array}$ & $\begin{array}{l}P_{T L}^{0}=1 ; P_{T L}^{g}=3 \\
x=0.3\end{array}$ \\
\hline $\begin{array}{l}\text { 1-LS parameters: none } \\
\text { PSO parameters }\end{array}$ & \\
\hline Swarm pop. size & $\mathcal{P}=30$ vector-candidates \\
\hline Veloc. bound factor & $V_{\max }=4$ \\
\hline Inertial weight & $\omega=1$ \\
\hline Acceleration coefficients & $\phi_{1}=2($ local $) ; \phi_{2}=2$ (global) \\
\hline \multicolumn{2}{|l|}{ Rayleigh channel } \\
\hline Max. Doppler freq. & $f_{\mathrm{Dpl}}=100 \mathrm{~Hz}$ (slow channel) \\
\hline Per subcarrier & Flat-frequency \\
\hline Channel state info. & Perfectly known at Rx and with errors \\
\hline
\end{tabular}

single-user bound $(\mathrm{SuB})$ is included in some performance plots. In all simulated systems, the time processing gain is $N=8$, and there are $M=4$ subcarriers, i.e., the equivalent processing gain is $N M=32$.

It is assumed perfect CSI estimation in all numerical results, except in Sect. 5.2, where channel estimation errors are modeled stochastically.

\subsection{Convergence and BER Performance}

First of all, it is important to highlight that the obtained Monte-Carlo simulation (MCS) results indicate that SA, PSO, STTS, RTS, GA, and 1-LS MUDs reach a similar performance after convergence, although with different computational complexity, as discussed in Sect. 4. Then, assuming different number of receive antennas, the BER performance of these algorithms, as a function of $E_{b} / N_{0} \in[0 ; 18] \mathrm{dB}$, is represented in Fig. 3 by the label "Heur", while "CD" indicates conventional detector performance. For medium (Fig. 3a) and full sys- 

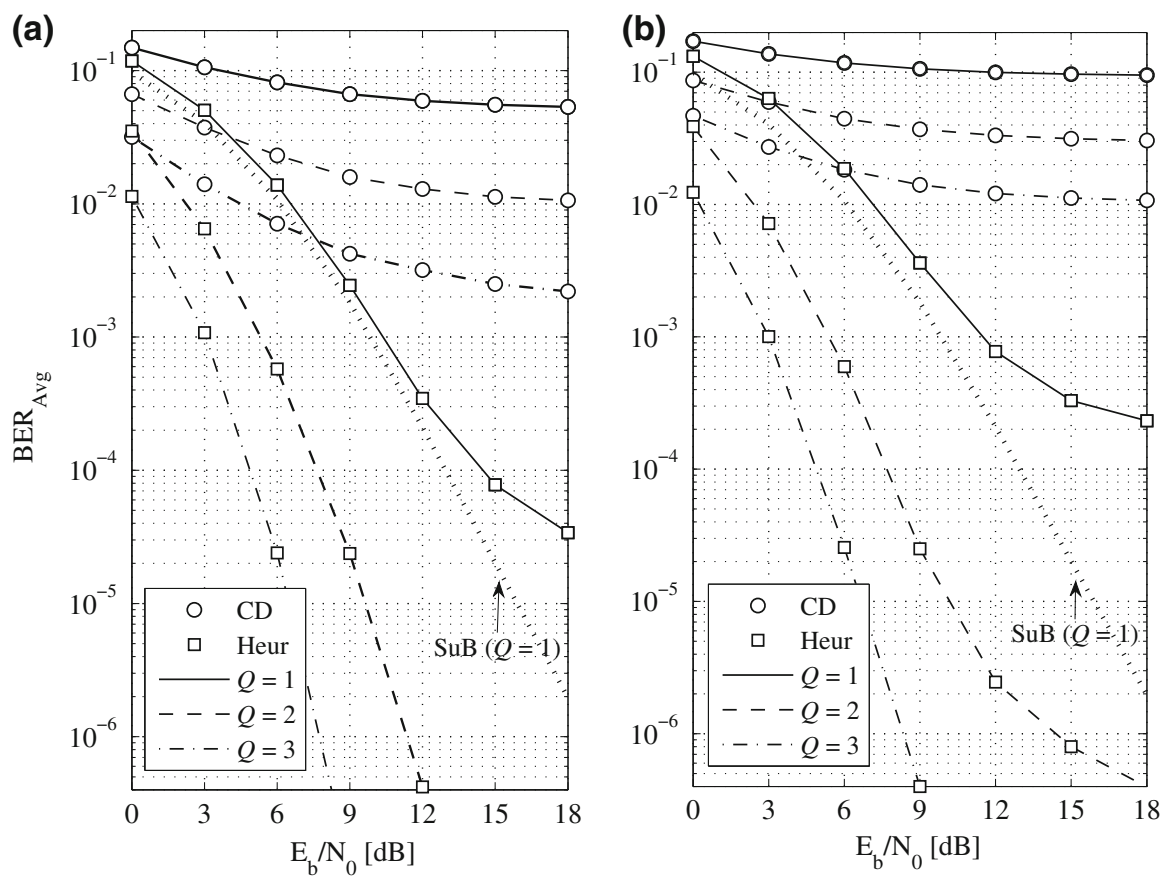

Fig. 3 BER performance of Heur-MuD as a function of $E_{b} / N_{0}$ in dB. a $K=20$, b $K=32$

tem loading ( $L=1$ ) (Fig. 3b), the MC-CDMA system equipped with one of the Heur-MuDs achieves a better BER performance than the CD. In both evaluated system loadings, it is observed a BER floor for $E_{b} / N_{0}>12 \mathrm{~dB}$ and $Q=1$. For $L=1$, such a BER floor is also noticed with $Q=2$ antennas. Moreover, mainly for $E_{b} / N_{0}>9 \mathrm{~dB}$, an increase in the number of users from $K=20$ to $K=32$ causes a BER performance degradation.

The performance of Heur-MuDs as a function of $K \in[2,32]$, for $E_{b} / N_{0}=3,6$ and $9 \mathrm{~dB}$, $Q=1$ and 2 antennas, is shown in Fig. 4. In these SNR conditions, an increase in the system loading causes a minor BER degradation. Such a degradation is more evident for $Q=1$. However, according to Fig. 3, for higher SNRs, it is expected a greater sensitivity regarding the system loading conditions.

To corroborate the assertion that after convergence all Heur-MUDs reach a similar BER performance, their convergence speed are selected and compared in Figs. 5 and 6, considering medium and full system loadings, perfect CSI estimation, and $E_{b} / N_{0}=6 \mathrm{~dB}$. For GA and PSO, the population size is set to $\mathcal{P}=30$. Note that, an increase in the number of users causes only a little BER degradation, but the convergence becomes slower, since the initial solution (CD) is worst. ${ }^{2}$

It is worth noticing that, in all convergence figures, 1-LS, STTS, RTS and SA have the same convergence speed, which is faster than GA and PSO.

2 With an increase in the system loading, the MAI effect is more evident, resulting in more bit errors to be corrected and, consequently, a longer search. 
(a)

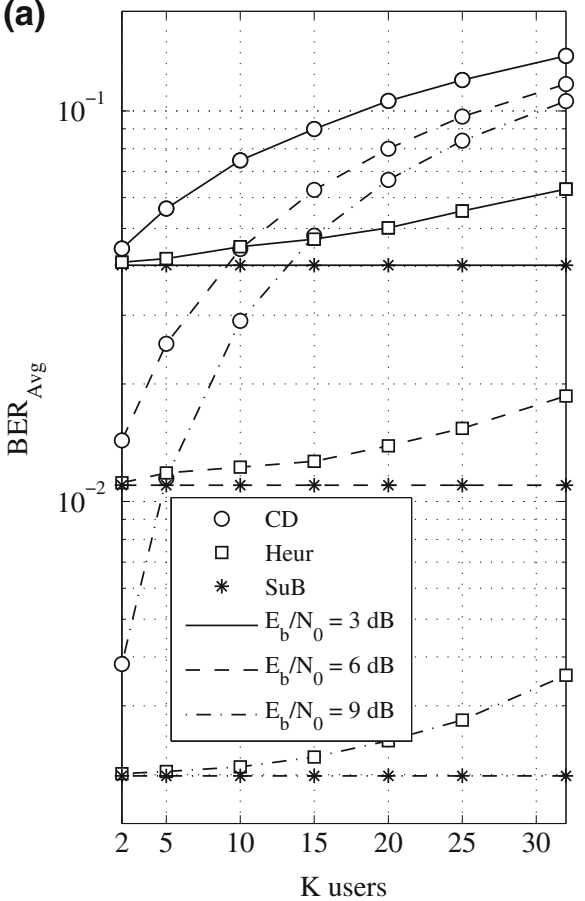

(b)

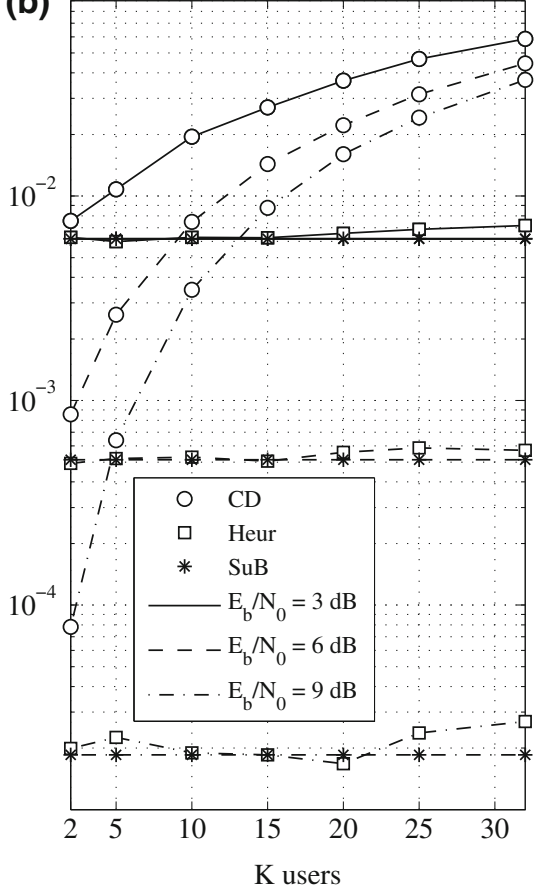

Fig. 4 BER performance of Heur-MuDs as a function of the number of users. $\mathbf{a} Q=1$ and $\mathbf{b} Q=2$ receive antennas

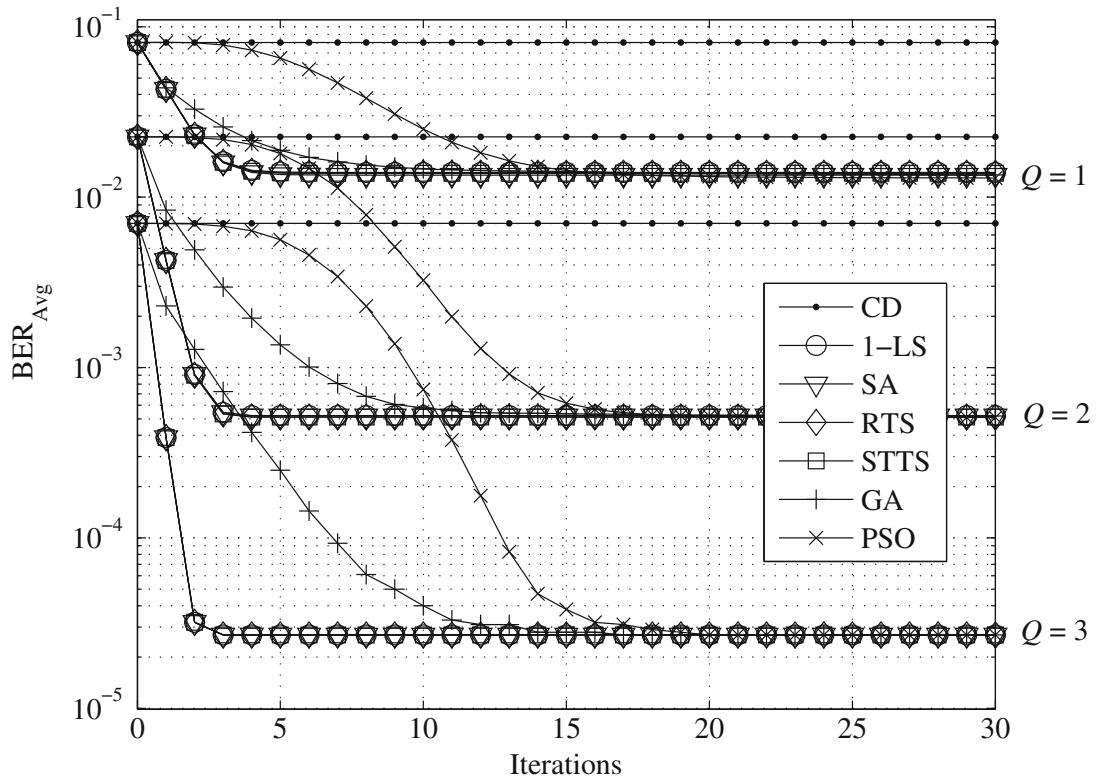

Fig. 5 Convergence performance of all Heur-MuDs with $E_{b} / N_{0}=6 \mathrm{~dB}, K=20, \mathcal{P}=30$ (GA and PSO) 


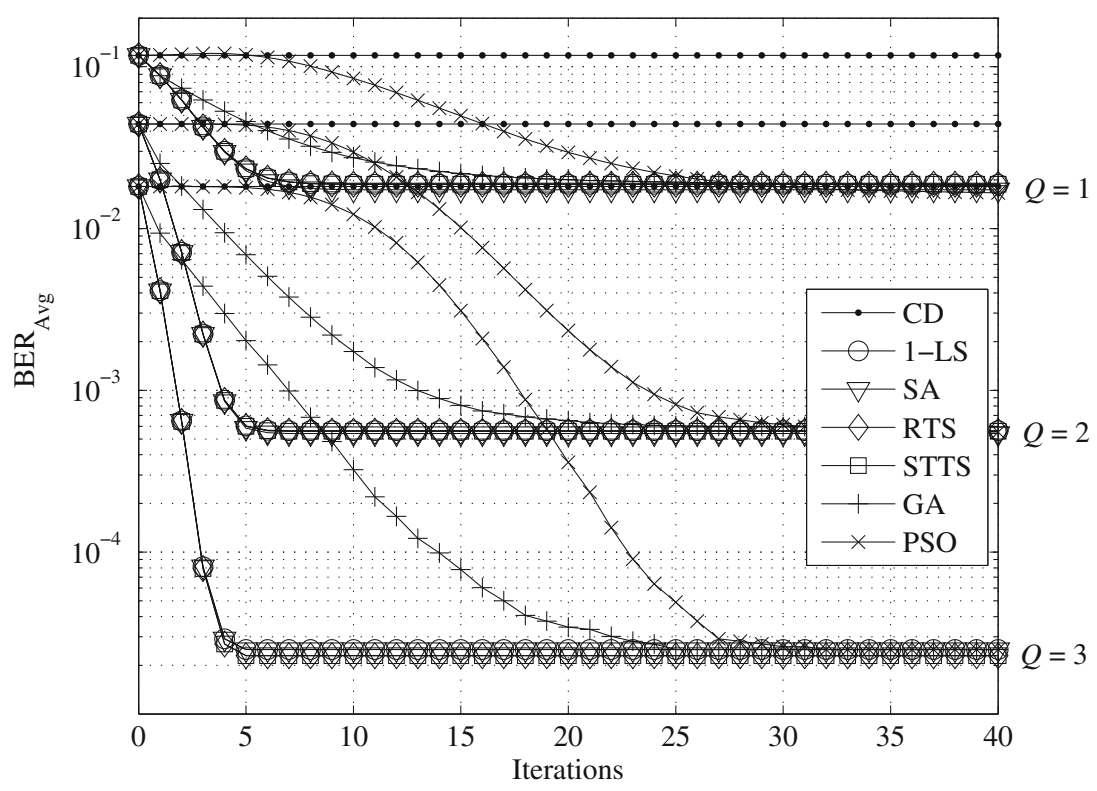

Fig. 6 Convergence performance of all Heur-MuDs with $E_{b} / N_{0}=6 \mathrm{~dB}, K=32, \mathcal{P}=30$ (GA and PSO)

\subsection{BER Performance Under CSI Errors Estimate}

In this subsection the impact of the channel estimation errors on the BER performance of the Heur-MuDs is analyzed. These errors are modeled stochastically using a continuous uniform distribution $^{3} \mathcal{U}[1 \pm \epsilon]$, centralized on the true coefficients values, resulting in

$$
\widehat{\beta}_{q, k, m}=\mathcal{U}\left[1 \pm \epsilon_{\beta}\right] \times \beta_{q, k, m} ; \quad \widehat{\varphi}_{q, k, m}=\mathcal{U}\left[1 \pm \epsilon_{\varphi}\right] \times \varphi_{q, k, m},
$$

where $\epsilon_{\beta}$ and $\epsilon_{\varphi}$ are the maximum normalized amplitude and phase errors for the channel coefficients, respectively.

Figure 7 shows the Heur-MuDs convergence for full system loading, considering CSI estimation errors modeled as (20), with $\epsilon_{\beta}=\epsilon_{\varphi}=5 \%$ and $15 \%$. Note from Fig. $7 \mathrm{c}$ that, even for $\epsilon_{\beta}=\epsilon_{\varphi}=15 \%$, the BER performance degradation is marginal when compared to the perfect CSI estimation case.

\subsection{Numerical Comparison of Computational Complexity}

Figure 8 presents the $\mathcal{P} G$ product as an representative complexity measure to guarantee convergence under distinct system loadings and number of receive antennas for all heuristic multiuser detectors. Note that, while the product $\mathcal{P} G$ for the unitary Hamming distance local search strategies shows a reduction trend when the number of receive antennas increases, the PSO and GA complexities mostly increase with an increment in $Q$. In addition, all Heur-MuD schemes exhibit an increment in the $\mathcal{P} G$ product when the system loading increases. Indeed, these two behaviors can be observed in Figs. 5, 6, and 7.

\footnotetext{
3 As a result, the probability density function is given by $f(x)=\left\{\begin{array}{ll}1 / 2 \epsilon, & (1-\epsilon) c \leq x \leq(1+\epsilon) c \\ 0, & \text { otherwise }\end{array}\right.$, where $c$ is the true value of either the module $\beta$, or the phase $\varphi$.
} 

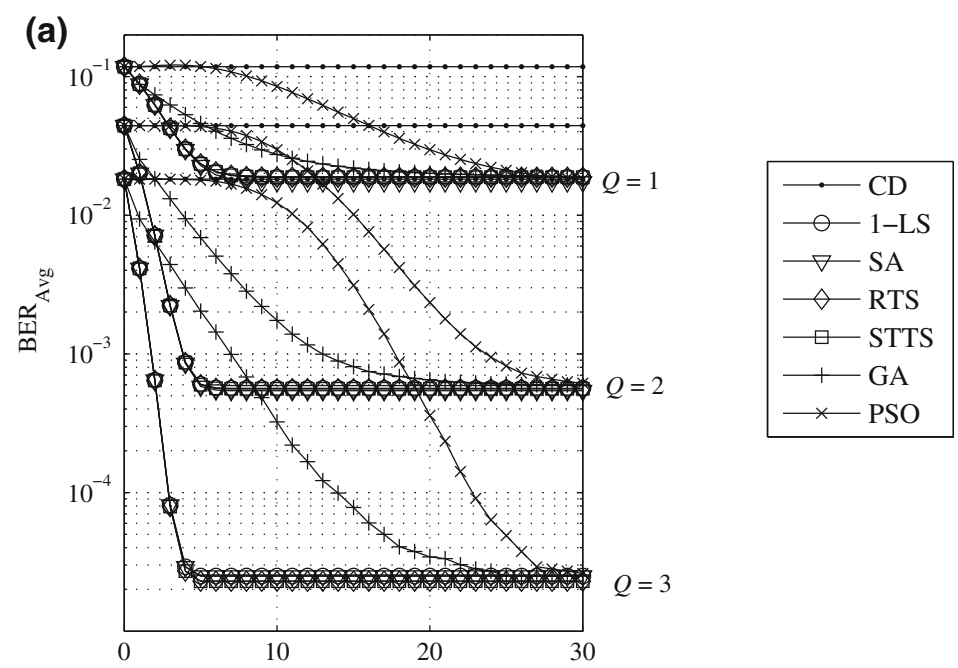

(b)

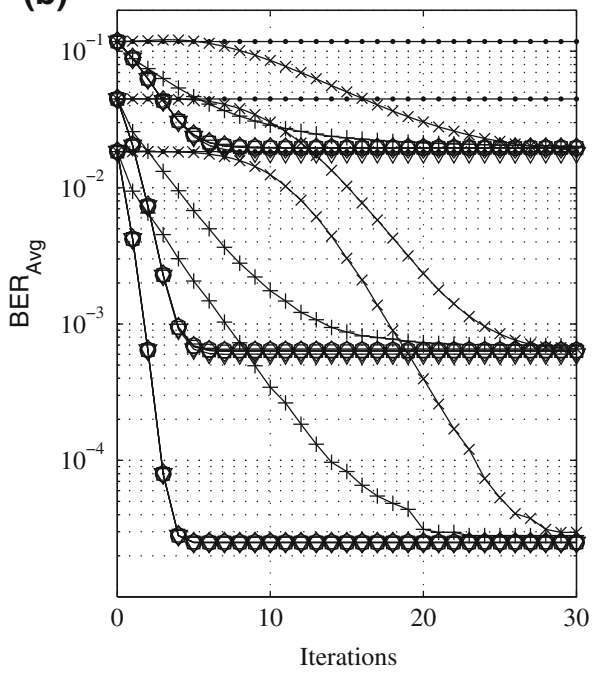

(c)

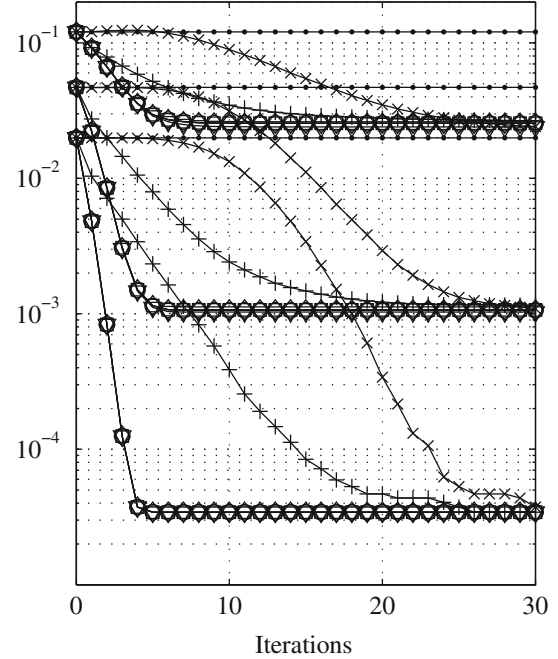

Fig. 7 Heur-MuDs convergence for $K=32$ users, $E_{b} / N_{0}=6 \mathrm{~dB}, \mathcal{P}=30$ (GA and PSO), and $\mathbf{a} \epsilon_{\beta}=$ $\epsilon_{\varphi}=0 \%$ (perfect CSI estimation), $\mathbf{b} \epsilon_{\beta}=\epsilon_{\varphi}=5 \%$, and $\mathbf{c} \epsilon_{\beta}=\epsilon_{\varphi}=15 \%$

Using the expressions presented in Table 2, Fig. 9 takes in perspective the complexity figures of merit considering perfect CSI estimation and the conditions described in Table 4. The CF factor decreases slightly with an increase in the number of antennas for all uH-ds strategies, resulting in approximately twice the one-CFC, while for the PSO and GA, the CF increases with $Q$. This trend indicates that PSO and GA algorithms may need some extra adjustment over input parameters (see Table 3), when the number of receive antennas and/or system loading change.

Besides, Figs. 9c and d show the complexity reduction (CR) index for $K=20$ (System I) and $K=32$ (System II). All the six Heur-MuDs achieve considerable reductions on the complexity when compared to the OMUD implementation (of the order of $10^{4}$ and $10^{7}$, for System I and II, respectively). Among these heuristic approaches, the uH-ds strategies represent the 


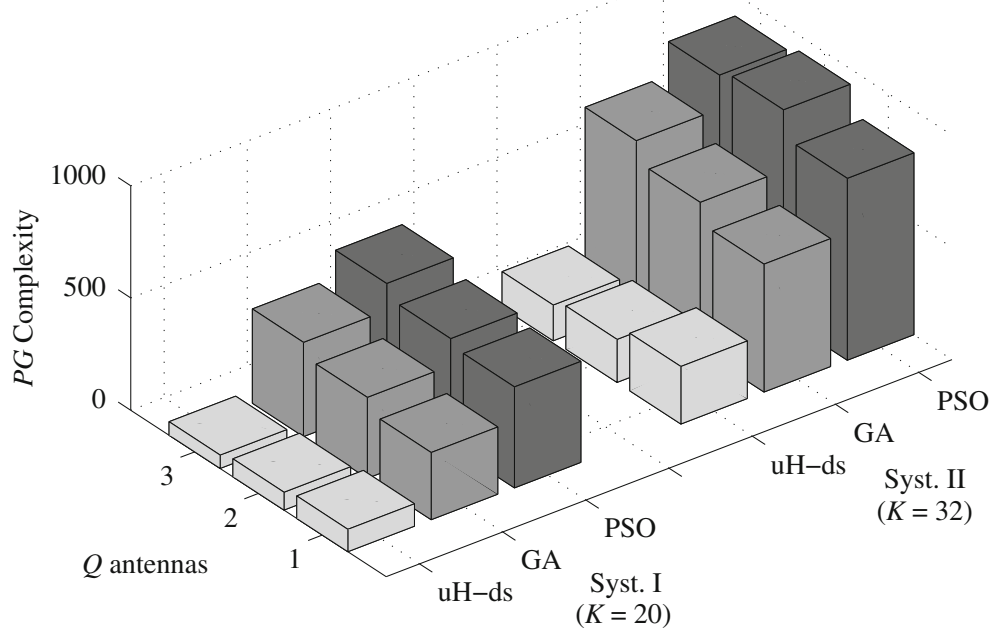

Fig. 8 Product $\mathcal{P} G$ for convergence of Heur-MuDs under two system loading conditions and $Q=1,2$ and 3 antennas

Table 4 Number of iterations for convergence $(\mathcal{G})$ under different conditions; $\mathcal{P}=30$ (GA and PSO)

\begin{tabular}{lll}
\hline$Q \in\left[\begin{array}{lll}1 & 23\end{array}\right]$ & Syst. I (20 us.) & Syst. II (32 us.) \\
\hline $\mathcal{G}_{\mathrm{uH}-\mathrm{ds}}$ & {$\left[\begin{array}{llll}5 & 4 & 3\end{array}\right]$} & {$\left[\begin{array}{lll}8 & 6 & 5\end{array}\right]$} \\
$\mathcal{G}_{\mathrm{GA}}$ & {$\left[\begin{array}{llll}10 & 12 & 14\end{array}\right]$} & {$\left[\begin{array}{lll}19 & 22 & 25\end{array}\right]$} \\
$\mathcal{G}_{\mathrm{PSO}}$ & {$\left[\begin{array}{lll}15 & 16 & 18\end{array}\right]$} & {$\left[\begin{array}{lll}27 & 31 & 30\end{array}\right]$} \\
Obs & Fig. 5 & Fig. 6 \\
\hline
\end{tabular}

best choice. Moreover, the 1-LS-MUD results in the best complexity-performance trade-off, with further advantages of absence of input parameters and simple stop criterion.

\section{Conclusions}

This work took in perspective six near-optimum heuristic multiuser detectors suitable for synchronous S/MIMO MC-CDMA systems. A single-objective antenna-diversity-aided strategy was considered. Based on extensive Monte-Carlo simulation results, an accurate performance-complexity trade-off analysis was carried out.

All the Heur-MUD approaches reached a similar BER performance with moderate complexity costs, evidencing the potentiality of these techniques in multiple-access wireless detection applications.

The complexities of uH-ds strategies are quite similar but substantially smaller than PSO and GA approaches, resulting in a complexity reduction factor of at least two times. This represents an implementation advantage of the unitary Hamming distance search strategies.

While under unitary Hamming distance search strategies the number of iterations for convergence decreases with an increase in the number of receive antennas, it is interesting to note the opposite tendency with the PSO and GA schemes.

Finally, results showed that 1-LS-MUD provides the best performance-complexity trade-off among all the Heur-MuD schemes analyzed, with additional advantages regarding the absence of input parameters and a simple stop criterion. 

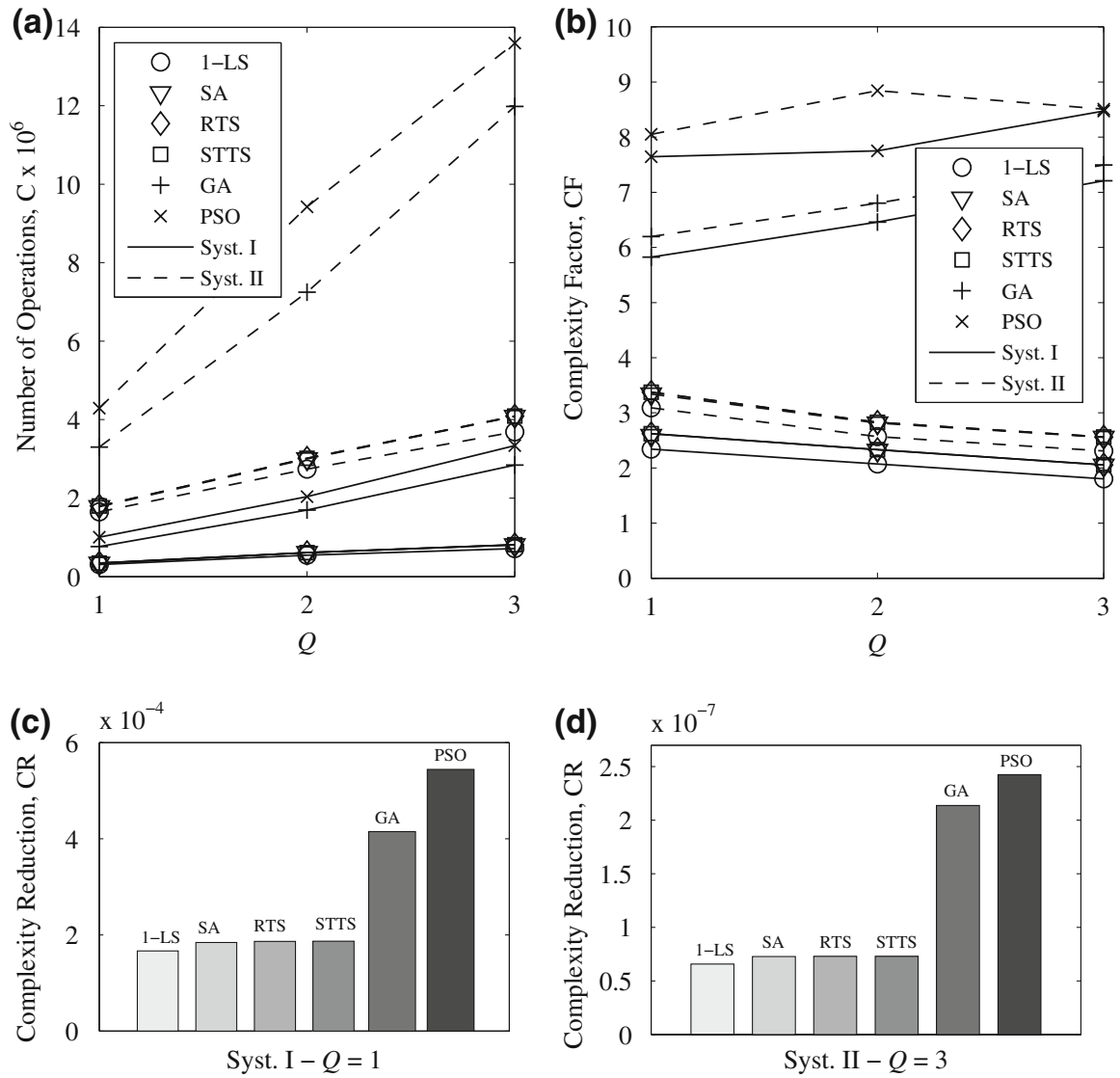

Fig. 9 Complexity indexes: a number of operations $\mathrm{C}, \mathbf{b}$ complexity factor $\mathrm{CF}, \mathbf{c}$ and $\mathbf{d}$ complexity reduction CR

Acknowledgements This work was partially supported by the Brazilian CAPES Agency Grants, under Fellowship code BEX0556/07-6.

Open Access This article is distributed under the terms of the Creative Commons Attribution Noncommercial License which permits any noncommercial use, distribution, and reproduction in any medium, provided the original author(s) and source are credited.

\section{Appendix}

Pseudo-Codes

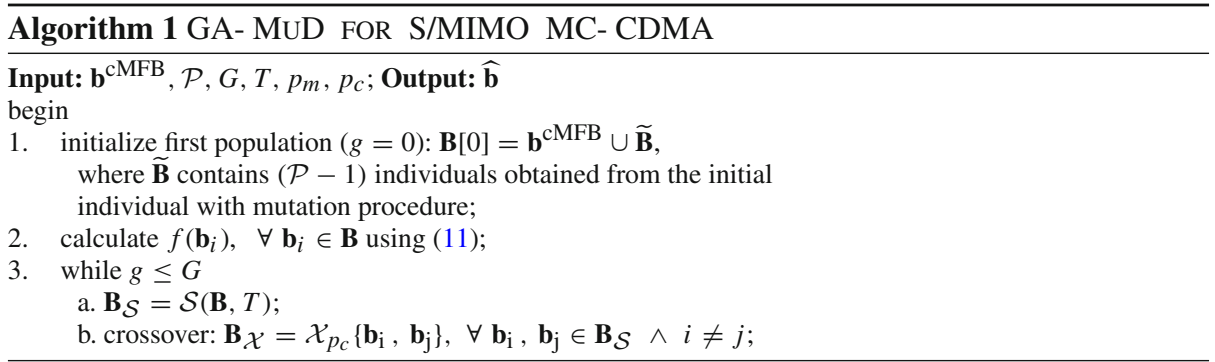



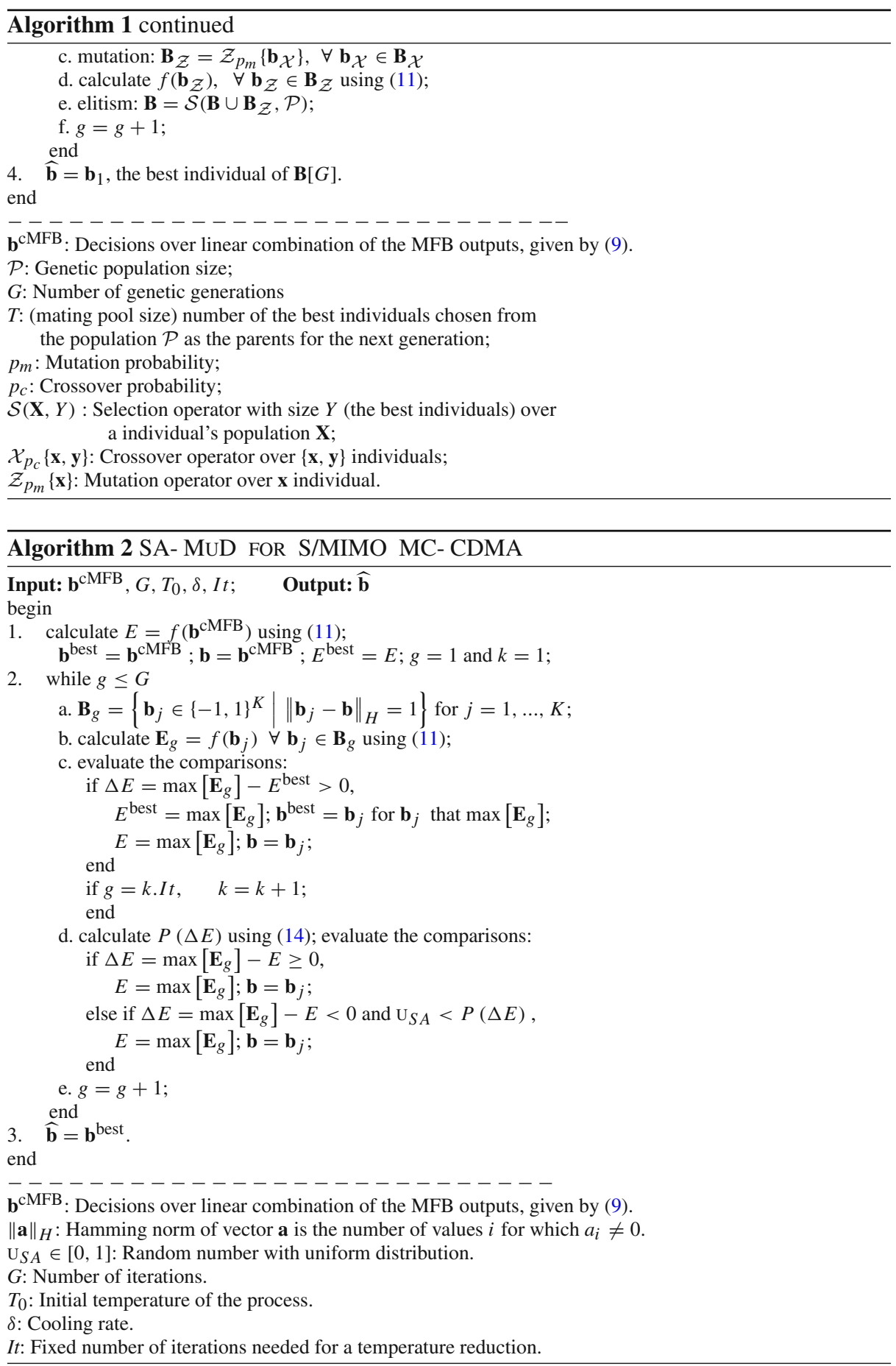


\section{Algorithm 3 STTS- MUD FOR S/MIMO MC- CDMA}

\section{Input: $\mathbf{b}^{\mathrm{cMFB}}, G, P_{T L} ; \quad$ Output: $\widehat{\mathbf{b}}$}

begin

1. calculate $E=f\left(\mathbf{b}^{\mathrm{cMFB}}\right)$ using (11);

$\mathbf{b}^{\text {best }}=\mathbf{b}^{\mathrm{cMFB}} ; \mathbf{b}=\mathbf{b}^{\mathrm{cMFB}} ; E^{\text {best }}=E ; g=1$;

$T_{a b u}^{0}$ List $=\oslash ;$ Aspiration $=0$ and Equal $=0$;

2. while $g \leq G$

a. $\mathbf{B}_{g}=\left\{\mathbf{b}_{j} \in\{-1,1\}^{K} \mid\left\|\mathbf{b}_{j}-\mathbf{b}\right\|_{H}=1\right\}$ for $j=1, \ldots, K$;

b. calculate $\mathbf{E}_{g}=f\left(\mathbf{b}_{j}\right) \forall \mathbf{b}_{j} \in \mathbf{B}_{g}$ using (11);

c. $C_{j}=\mathbf{b}_{j} \oplus \mathbf{b}$ for $\mathbf{b}_{j}$ that $\max \left[\mathbf{E}_{g}\right]$;

d. evaluate the comparisons:

$$
\text { if } T a b u_{\text {List }}^{g-1} \neq \oslash \text {, }
$$

while Aspiration $=0$,

if $C_{j} \in T a b u_{\text {List }}^{g-1}, \quad$ Equal $=1$;

else, $\quad$ Equal $=0$;

end

if Equal $\neq 0$,

if $\max \left[\mathbf{E}_{g}\right]>E^{\text {best }}, \quad$ Aspiration $=1$;

else, $\quad$ Aspiration $=0$;

end

else, $\quad$ Aspiration $=1$;

end

end

end

Aspiration $=0$;

if $\max \left[\mathbf{E}_{g}\right]>E^{\text {best }}$,

$\mathbf{b}^{\text {best }}=\mathbf{b}_{j}$ and $\mathbf{b}=\mathbf{b}_{j}$;

$E^{\text {best }}=\max \left[\mathbf{E}_{g}\right]$ and $E=\max \left[\mathbf{E}_{g}\right]$;

Aspiration $=1$;

else if $\max \left[\mathbf{E}_{g}\right]>E$,

$\mathbf{b}=\mathbf{b}_{j}$ and $E=\max \left[\mathbf{E}_{g}\right]$

e. Update the tabu list: $\operatorname{Tabu}_{\text {List }}^{g}=\left[\begin{array}{lll}T a b u_{\text {List }}^{g-1}, C_{j} & ] \text {; }\end{array}\right.$

f. if length $\left[T a b u_{L i s t}^{g}\right] \geq P_{T L}$, (comparison) end

$T a b u_{\text {List }}^{g}$ is emptied and Equal $=0$;

g. $g=g+1$;

3. $\widehat{\mathbf{b}}=\mathbf{b}^{\text {best }}$.

end

b $^{\mathrm{cMFB}}$ : Decisions over linear combination of the MFB outputs, given by (9).

$\|\mathbf{a}\|_{H}$ : Hamming norm of vector $\mathbf{a}$ is the number of values $i$ for which $a_{i} \neq 0$.

$G$ : Number of iterations.

$P_{T L}$ : Fixed prohibition period.

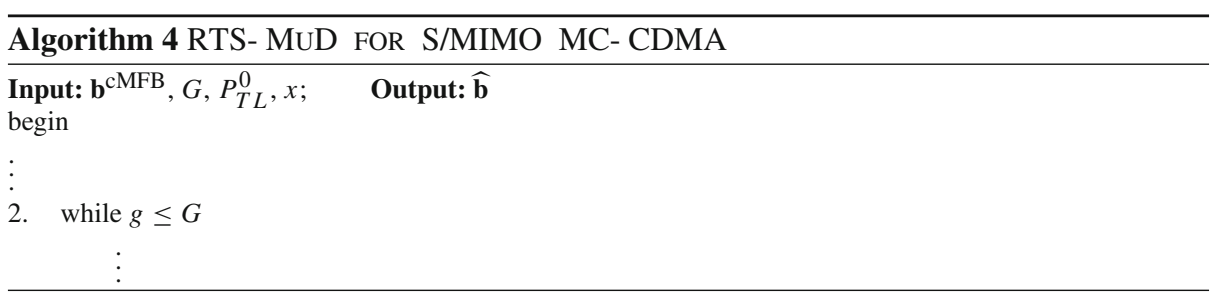



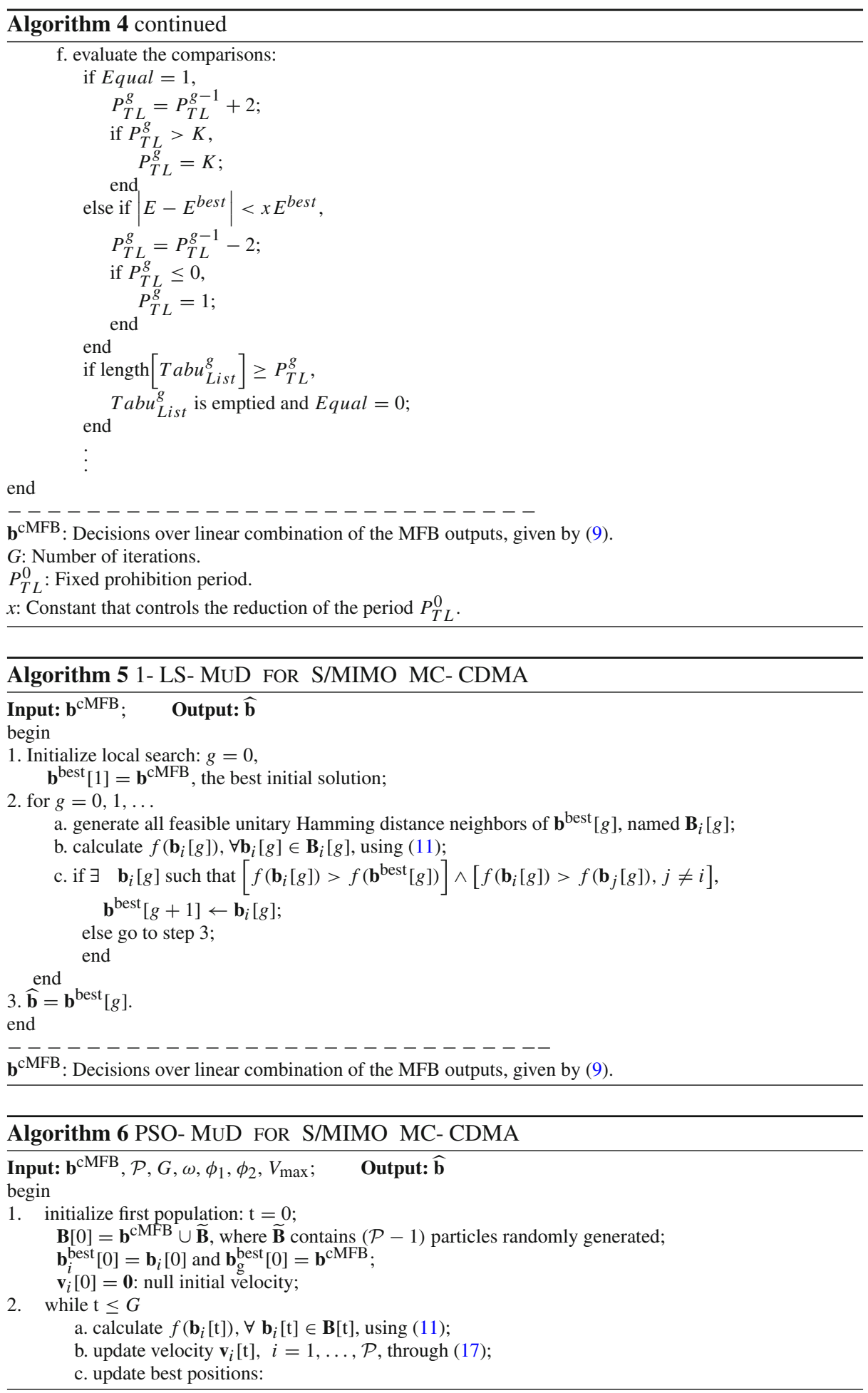


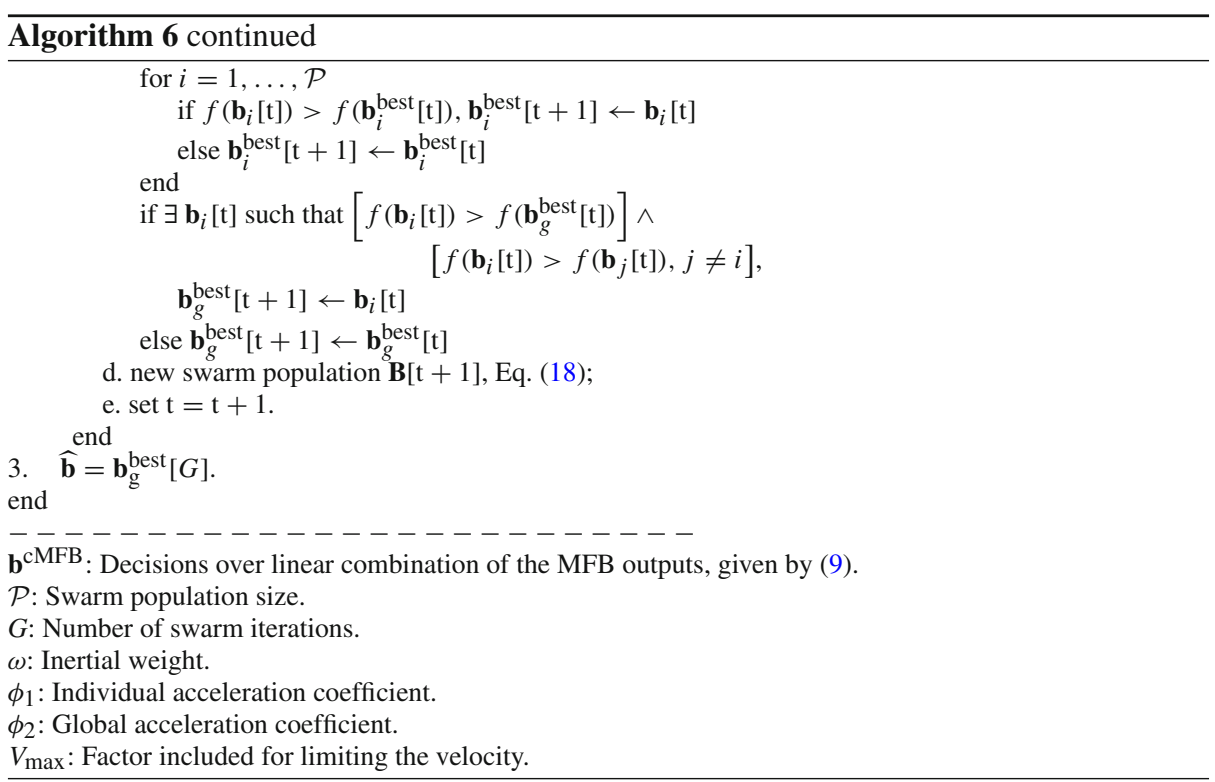

\section{References}

1. Aarts, E. H. L., \& Lenstra, J. K. (2003). Local search in combinatorial optimization (536 pp). USA : Princeton University Press.

2. Abrão, T., Ciriaco, F., \& Jeszensky, P. J. E. (2004). Evolutionary programming with cloning and adaptive cost function applied to multi-user ds-cdma systems. In ISSSTA'04 (pp. 160-163), Sydney, Australia.

3. Abrão, T., Ciriaco, F., Oliveira, L. D., Angélico, B. A., Jeszensky, P. J. E., \& Casadevall, F. (2008). Weighting particle swarm optimization simo mc-cdma multiuser detectors. In ISSSTA'08-10th international symposium on spread spectrum techniques and applications (pp. 69-73). Bologna, Italy.

4. Bashir, S., Khan, A. A., Naeem, M., \& Shah, S. I. (2007). An application of ga for symbol detection in mimo communication systems. In Third international conference on natural computation ICNC 2007 (Vol. 2, pp. 404 - 410). Haikou.

5. Battiti, R., \& Tecchioli, G. (1994). The reactive tabu search. ORSA, Journal of Computing, 6, 126-140.

6. Cerny, V. (1985). Thermodynamical approach to the traveling salesman problem: An efficient simulation algorithm. Journal of Optimization theory and Application, 45, 41-51.

7. Ciriaco, F., Abrão, T., \& Jeszensky, P. J. E. (2006). Ds/cdma multiuser detection with evolutionary algorithms. Journal Of Universal Computer Science, 12(4), 450-480.

8. Ergün, C., \& Hacioglu, K. (2000). Multiuser detection using a genetic algorithm in cdma communications systems. IEEE Transactions on Communications, 48, 1374-1382.

9. Glover, F. (1977). Heuristic for integer programming using surrogate contraints. Decision Sciences, 8, $156-166$.

10. Glover, F. (1986). Future paths for integer programming and links to artificial intelligence. Computers \& Operations Research, 13, 533-549.

11. Glover, F., \& Laguna, M. (1997). Tabu search. Boston: Kluwer Academic Publishers.

12. Goldberg, D. E. (1989). Genetic algorithms in search, optimization, and machine learning. Reading, MA: Addison-Wesley.

13. Hara, S., \& Prasad, R. (1997). Overview of multicarrier CDMA. IEEE Communications Magazine, 35(12), 126-133.

14. Jalden, J., \& Ottersten, B. (2005). On the complexity of sphere decoding in digital communications. IEEE Transactions on Signal Processing, 53(4), 1474-1484.

15. Jiang, M., \& Hanzo, L. (2007). Multiuser mimo-ofdm for next-generation wireless systems. Proceedings of the IEEE, 95(7), 1430-1469. 
16. Jiang, Y., Zhong, Z., Yang, J., \& Zhang, M. (2007). Sub-optimal multiuser detector using a time-varying gain chaotic simulated annealing neural network. In ICNC 2007-Third international conference on natural computation (Vol. 1, pp. 305-309). Haikou.

17. Katayama, K., \& Narihisa, H. (2001). Performance of simulated annealing-based heuristic for unconstrained binary quadratic programming problem. European Journal of Operational Research, 134, $103-119$.

18. Kennedy, J., \& Eberhart, R. (1995). Particle swarm optimization. In IEEE international conference on neural networks (pp. 1942-1948).

19. Khan, A., Bashir, S., Naeem, M., \& Shah, S. (2006). Heuristics assisted detection in high speed wireless communication systems. In IEEE multitopic conference (pp. 1-5). Islamabad.

20. Kirkpatrick, S., Gellat, C. D., \& Vecchi, M. P. (1983). Optimization by simulated annealing. Science, 220, 671-680.

21. Lim, H. S., \& Venkatesh, B. (2003). An efficient local search heuristics for asynchronous multiuser detection. IEEE Communications Letters, 7(6), 299-301.

22. Ma, W. K., Davidson, T. N., Wong, K. M., Luo, Z. Q., \& Ching, P. C. (2002). Quasi-maximum-likelihood multiuser detection using semi-definite relaxation with applications to synchronous cdma. IEEE Transactions on Signal Processing, 50(4), 912-922 .

23. Mitchell, M. (1998). An introduction to genetic algorithms. Cambridge: MIT Press.

24. Mobasher, A., Taherzadeh, M., Sotirov, R., \& Khandani, A. K. (2005). A near maximum likelihood decoding algorithm for mimo systems based on semi-definite programming. In ISIT 2005-International symposium on information theory (pp. 1686-1690). Adelaide, SA.

25. Moshavi, S. (1996). Multi-user detection for ds-cdma communications. IEEE Communication Magazine, 34, 132-136.

26. Oliveira, L. D., Ciriaco, F., Abrão, T., \& Jeszensky, P. J. E. (2009). Local search multiuser detection. $A E \ddot{U}$ International Journal of Electronics and Communications, 63(4), 259-270.

27. Oliveira, L. D., Ciriaco, F., Abrão, T., \& Jeszensky, P. J. E. (2006). Particle swarm and quantum particle swarm optimization applied to ds/cdma multiuser detection in flat rayleigh channels. In ISSSTA'06IEEE international symposium on spread spectrum techniques and applications (pp. 133-137). Manaus, Brazil.

28. Oliveira, L. D., Ciriaco, F., Abrão, T., \& Jeszensky, P. J. E. (2007). Simplified local search algorithm for multiuser detection in multipath rayleigh channels. In The 16th IST mobile and wireless communications summit (pp. 1-4). Budapest, Hungary.

29. Press, W. H., Teukolsky, S. A., Vetterling, W. T., \& Flannery, B. P. (1992). Numerical recipes in C: The art of scientifc computing. Cambridge: Cambridge University Press.

30. Tan, P. H. (2001). Multiuser detection in cdma-combinatorial optimization methods. Master's thesis, Chalmers University of Technology, Göteborg.

31. Tan, P. H., \& Rasmussen, L. K. (2001). The application of semidefinite programming for detection in cdma. IEEE Journal on Selected Areas in Communication, 19(8), 1442-1449.

32. Vardhan, K. V., Mohammed, K., Chockalingan, A., \& Rajan, B. S. (2008). A low-complexity detector for large mimo systems and multicarrier cdma systems. IEEE Journal on Selected Areas in Communication, 26(3), 473-485.

33. Verdú, S. (1998). Multiuser Detection. New York: Cambridge University Press.

34. Wang, X. M., Lu, W. S., \& Antoniou, A. (2003). A near-optimal multiuser detector for ds-cdma using semidefinite programming relaxation. IEEE Transactions on Signal Processing, 51(9), 2446-2450.

35. Wei, H., \& Hanzo, L. (2004). Reduced-complexity near-optimum genetic algorithm assisted multiuser detection for synchronous multicarrier cdma. In IEEE 59th vehicular technology conference (Vol. 3, pp. 1717-1721).

36. Yen, K., \& Hanzo, L. (2003). Antenna-diversity-assisted genetic-algorithm-based multiuser detection schemes for synchronous cdma systems. IEEE Transactions On Communications, 51(3), 366-370.

37. Zhao, H., Long, H., \& Wang, W. (2006). Pso selection of surviving nodes in qrm detection for mimo systems. In GLOBECOM-IEEE global telecommunications conference (pp. 1-5). San Francisco, CA.

38. Zhao, Y., \& Zheng, J. (2004). Particle swarm optimization algorithm in signal detection and blind extraction. In IEEE (Ed.), 7th international symposium on parallel architectures, algorithms and networks (pp. 37-41).

39. Zong, P., Wang, K., \& Bar-Ness, Y. (2001). Partial sampling mmse interference suppression in asynchronous mc-cdma systems. IEEE Journal on Selected Areas in Communication, 9(8), 1605-1613. 


\section{Author Biographies}
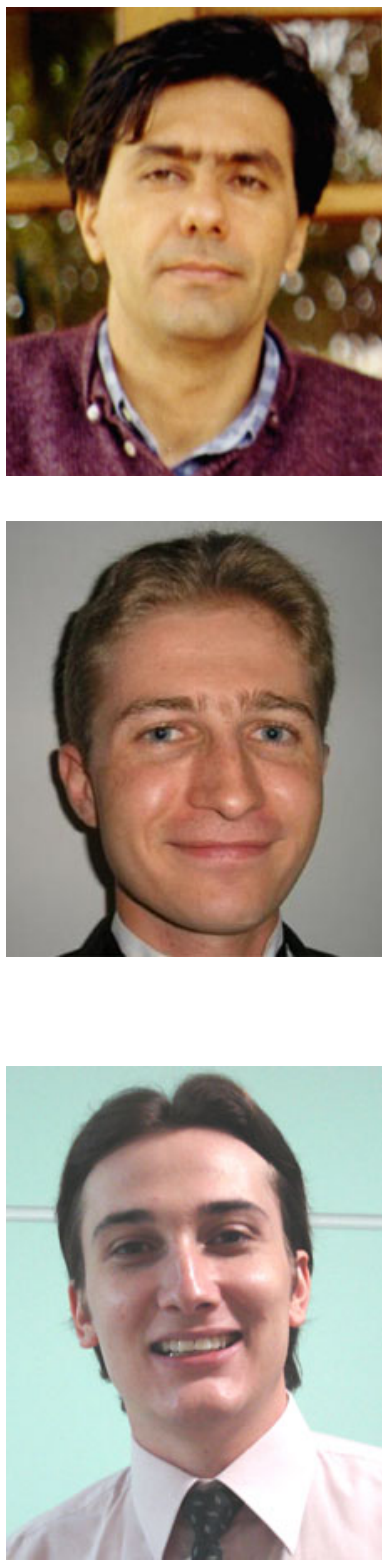

Taufik Abrão received the B.S., M.Sc. and Ph.D., all in Electrical Engineering from EPUSP-Escola Politécnica of University São Paulo, Brazil, in 1992, 1996, and 2001, respectively. He is currently an Adjunct Professor at the Electrical Engineering Department of UELState University of Londrina (Brazil). Currently (2007/2-2008/1) he is a visiting professor at TSC/UPC_-Departament de Teoria del Senyal i Comunicacions, Universitat Politécnica de Catalunya, Barcelona, Spain. His research interests include multi-user detection, MC-CDMA and MIMO systems, heuristic and optimization aspects of DS-CDMA systems and $4 \mathrm{G}$ systems. He is author or co-author of more than 60 research papers published in specialized periodicals and symposiums.

Leonardo D. de Oliveira received the B.S. and M.Sc degrees in Electrical Engineering from UEL-State University of Londrina (Brazil) in 2005 and 2007, respectively. He is currently a Ph.D. student at EPUSP - Escola Politécnica of University of São Paulo (Brazil), sponsored by QUALCOMM. His research interests include multiuser detection, MIMO, channel estimation and Ultra Wideband Systems.

Fernando Ciriaco received the B.S. and M.Sc degrees in Electrical Engineering from UEL-State University of Londrina (Brazil) in 2004 and 2006, respectively. He is currently a Ph.D. student at EPUSPEscola Politécnica of University of São Paulo (Brazil) and an Assistant professor at the Electrical Engineering Department of UEL and at the Faculdade Pitágoras (Brazil). His research interests are multiuser detection, heuristic algorithms, MIMO, MC-CDMA systems and wireless communications. 

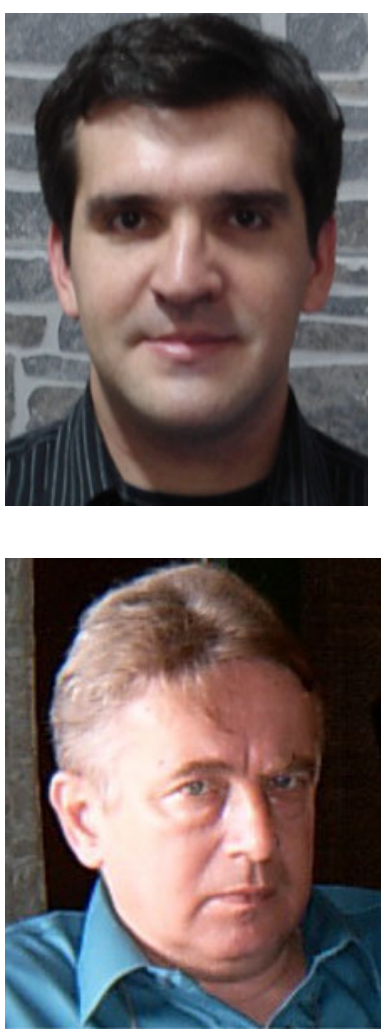

Bruno A. Angélico received the B.S. degree in Electrical Engineering from State University of Londrina (UEL), Brazil, and M.S. in Electrical Engineering from Escola Politécnica of University of São Paulo (EPUSP), Brazil. He is currently a Ph.D. student in Electrical Engineering at EPUSP. His research interests include ultra wideband systems, adaptive filters, multi-user detection and MIMO Systems.

Paul Jean E. Jeszensky received the B.S., M.S. and Ph.D., all in Electrical Engineering from EPUSP-Escola Politécnica of University of São Paulo (Brazil), in 1972, 1981, and 1989, respectively. Since 1990 he has been with EPUSP where he is Full Professor and Researcher in Communication Systems. He was visiting professor at UPC-Universitat Politécnica de Catalunya, Barcelona (Spain) in 1995 and at TUBTechnical University of Budapest (Hungary) in 2001. He is author of the book Sistemas Telefônicos (in Portuguese), Editora Manole, 2003 and his current research interests include CDMA systems, multi-user detection, code sequences analysis and related topics.

Fernando Jose Casadevall Palacio received the Engineer of Telecommunication and Dr Engineering degrees from the Universitat Politécnica de Catalunya (UPC), Spain, in 1977 and 1983 respectively. He is currently Full Professor in the Signal Theory and Communications Department. After graduation he was concerned with equalisation techniques for digital fibre optic systems. He has also been working in the field of digital communications with particular emphasis on digital radio and its performance under multipath propagation conditions. In the last ten years, he has mainly been concerned with the performance analysis and development of digital mobile radio systems. In particular his research interest include cellular and personal communication system, multipath transceiver design (including Software Radio techniques), Mobility and Radio Resources Management, End to end QoS issues. During the last ten years he participated in more than twenty research-projects founded by both public and private organisations. He has published around one hundred of technical papers in both International Conferences and Magazines. 